\title{
New Interior Penalty Discontinuous Galerkin Methods for the Keller-Segel Chemotaxis Model
}

\author{
Yekaterina Epshteyn* and Alexander Kurganov ${ }^{\dagger}$
}

\begin{abstract}
We develop a family of new interior penalty discontinuous Galerkin methods for the Keller-Segel chemotaxis model. This model is described by a system of two nonlinear PDEs: a convection-diffusion equation for the cell density coupled with a reaction-diffusion equation for the chemoattractant concentration. It has been recently shown that the convective part of this system is of a mixed hyperbolic-elliptic type, which may cause severe instabilities when the studied system is solved by straightforward numerical methods. Therefore, the first step in the derivation of our new methods is made by introducing the new variable for the gradient of the chemoattractant concentration and by reformulating the original Keller-Segel model in the form of a convection-diffusion-reaction system with a hyperbolic convective part. We then design interior penalty discontinuous Galerkin methods for the rewritten Keller-Segel system. Our methods employ the central-upwind numerical fluxes, originally developed in the context of finite-volume methods for hyperbolic systems of conservation laws.

In this paper, we consider Cartesian grids and prove error estimates for the proposed high-order discontinuous Galerkin methods. Our proof is valid for pre-blow-up times since we assume boundedness of the exact solution. We also show that the blow-up time of the exact solution is bounded from above by the blow-up time of our numerical solution. In the numerical tests presented below, we demonstrate that the obtained numerical solutions have no negative values and are oscillation-free, even though no slope limiting technique has been implemented.
\end{abstract}

AMS subject classification: 65M60, 65M12, 65M15, 92C17, 35K57

Key words: Keller-Segel chemotaxis model, convection-diffusion-reaction systems, discontinuous Galerkin methods, NIPG, IIPG, and SIPG methods, Cartesian meshes.

\section{Introduction}

The goal of this work is to design new Discontinuous Galerkin (DG) methods for the twodimensional (2-D) Keller-Segel chemotaxis model, [13, 28, 29, 30, 35, 37]. The DG methods have recently become increasingly popular thanks to their attractive features such as:

- local, element-wise mass conservation;

*Department of Mathematical Sciences, Carnegie Mellon University, Pittsburgh, PA, 15213, rina10Qandrew. cmu .edu

${ }^{\dagger}$ Mathematics Department, Tulane University, New Orleans, LA 70118; kurganov@math.tulane.edu 
- flexibility to use high-order polynomial and non-polynomial basis functions;

- ability to easily increase the order of approximation on each mesh element independently;

- ability to achieve almost exponential convergence rate when smooth solutions are captured on appropriate meshes;

- block diagonal mass matrices, which are of great computational advantage if an explicit time integration is used;

- suitability for parallel computations due to (relatively) local data communications;

- applicability to problems with discontinuous coefficients and/or solutions;

- The DG methods have been successfully applied to a wide variety of problems ranging from the solid mechanics to the fluid mechanics (see, e.g., $[3,7,14,15,17,20,22,40]$ and references therein).

In this paper, we consider the most common formulation of the Keller-Segel system [13], which can be written in the dimensionless form as

$$
\left\{\begin{array}{l}
\rho_{t}+\nabla \cdot(\chi \rho \nabla c)=\Delta \rho, \quad(x, y) \in \Omega, t>0 \\
c_{t}=\Delta c-c+\rho
\end{array}\right.
$$

subject to the Neumann boundary conditions:

$$
\nabla \rho \cdot \mathbf{n}=\nabla c \cdot \mathbf{n}=0, \quad(x, y) \in \partial \Omega
$$

Here, $\rho(x, y, t)$ is the cell density, $c(x, y, t)$ is the chemoattractant concentration, $\chi$ is a chemotactic sensitivity constant, $\Omega$ is a bounded domain in $\mathbb{R}^{2}, \partial \Omega$ is its boundary, and $\mathbf{n}$ is a unit normal vector.

It is well-known that solutions of this system may blow up in finite time, see, e.g., [26, 27] and references therein. This blow-up represents a mathematical description of a cell concentration phenomenon that occurs in real biological systems, see, e.g., [1, 8, 10, 11, 16, 38].

Capturing blowing up solutions numerically is a challenging problem. A finite-volume, [21], and a finite-element, [34], methods have been proposed for a simpler version of the Keller-Segel model,

$$
\left\{\begin{array}{l}
\rho_{t}+\nabla \cdot(\chi \rho \nabla c)=\Delta \rho \\
\Delta c-c+\rho=0
\end{array}\right.
$$

in which the equation for concentration $c$ has been replaced by an elliptic equation using an assumption that the chemoattractant concentration $c$ changes over much smaller time scales than the density $\rho$. A fractional step numerical method for a fully time-dependent chemotaxis system from [41] has been proposed in [42]. However, the operator splitting approach may not be applicable when a convective part of the chemotaxis system is not hyperbolic, which is a generic situation for the original Keller-Segel model as it was shown in [12], where the finitevolume Godunov-type central-upwind scheme was derived for (1.1) and extended to some other chemotaxis and haptotaxis models.

The starting point in the derivation of the central-upwind scheme in [12] was rewriting the original system (1.1) in an equivalent form, in which the concentration equation is replaced with the corresponding equation for the gradient of $c$ :

$$
\left\{\begin{array}{l}
\rho_{t}+\nabla \cdot(\chi \rho \mathbf{w})=\Delta \rho, \\
\mathbf{w}_{t}-\nabla \rho=\Delta \mathbf{w}-\mathbf{w},
\end{array} \quad \mathbf{w} \equiv(u, v):=\nabla c .\right.
$$


This form can be considered as a convection-diffusion-reaction system

$$
\mathbf{U}_{t}+\mathbf{f}(\mathbf{U})_{x}+\mathbf{g}(\mathbf{U})_{y}=\Delta \mathbf{U}+\mathbf{r}(\mathbf{U}),
$$

where $\mathbf{U}:=(\rho, u, v)^{T}, \mathbf{f}(\mathbf{U}):=(\chi \rho u,-\rho, 0)^{T}, \mathbf{g}(\mathbf{U}):=(\chi \rho v, 0,-\rho)^{T}$, and $\mathbf{r}(\mathbf{U}):=(0,-u,-v)^{T}$. The system (1.2) is an appropriate form of the chemotaxis system if one wants to solve it numerically by a finite-volume method. Even though the convective part of the system (1.2) is not hyperbolic, some stability of the resulting central-upwind scheme was ensured by proving its positivity preserving property, see [12].

A major disadvantage of the system (1.2) is a mixed type of its convective part. When a high-order numerical method is applied to (1.2), a switch from a hyperbolic region to an elliptic one may cause severe instabilities in the numerical solution since the propagation speeds in the elliptic region are infinite. Therefore, in order to develop high-order DG methods for (1.1), we rewrite it in a different form, which is suitable for DG settings:

$$
\begin{aligned}
& \rho_{t}+(\chi \rho u)_{x}+(\chi \rho v)_{y}=\Delta \rho, \\
& c_{t}=\Delta c-c+\rho, \\
& u=c_{x}, \\
& v=c_{y},
\end{aligned}
$$

where the new unknowns $\rho, c, u, v$ satisfy the following boundary conditions:

$$
\nabla \rho \cdot \mathbf{n}=\nabla c \cdot \mathbf{n}=(u, v)^{T} \cdot \mathbf{n}=0, \quad(x, y) \in \partial \Omega .
$$

The new system (1.3)-(1.6) may also be considered as a system of convection-diffusion-reaction equations

$$
k \mathbf{Q}_{t}+\mathbf{F}(\mathbf{Q})_{x}+\mathbf{G}(\mathbf{Q})_{y}=k \Delta \mathbf{Q}+\mathbf{R}(\mathbf{Q}),
$$

where $\mathbf{Q}:=(\rho, c, u, v)^{T}$, the fluxes are $\mathbf{F}(\mathbf{Q}):=(\chi \rho u, 0,-c, 0)^{T}$ and $\mathbf{G}(\mathbf{Q}):=(\chi \rho v, 0,0,-c)^{T}$, the reaction term is $\mathbf{R}(\mathbf{Q}):=(0, \rho-c,-u,-v)$, the constant $k=1$ in the first two equations in (1.8), and $k=0$ in the third and the fourth equations there. As we show in $\S 3$, the convective part of the system (1.8) is hyperbolic.

In this paper, we develop a family of high-order DG methods for the system (1.8). The proposed methods are based on three primal DG methods: the Nonsymmetric Interior Penalty Galerkin (NIPG), the Symmetric Interior Penalty Galerkin (SIPG), and the Incomplete Interior Penalty Galerkin (IIPG) methods, [4, 18, 19, 39]. The numerical fluxes in the proposed DG methods are the fluxes developed for the semidiscrete finite-volume central-upwind schemes in [32] (see also $[31,33]$ and references therein). These schemes belong to the family of non-oscillatory central schemes, which are highly accurate and efficient methods applicable to general multidimensional systems of conservation laws and related problems. Like other central fluxes, the central-upwind ones are obtained without using (approximate) Riemann problem solver, which is unavailable for the system under consideration. At the same time, a certain upwinding information-one-sided speeds of propagation - is incorporated into the central-upwind fluxes.

We consider Cartesian grids and prove the error estimates for the proposed high-order DG methods under the assumption of boundedness of the exact solution. We also show that the blow-up time of the exact solution is bounded from above by the blow-up time of the solution of our DG methods. In numerical tests presented in $\S 6$, we demonstrate that the obtained numerical 
solutions have no negative values and are oscillation-free, even though no slope limiting technique has been implemented. We also demonstrate a high order of numerical convergence, achieved even when the final computational time gets close to the blowup time and the spiky structure of the solution is well developed.

The paper is organized as follows. In $\S 2$, we introduce our notations and assumptions, and state some standard results. The new DG methods are presented in $\S 3$. The consistency and error analysis of the proposed methods are established in Sections 4 and 5 (some proof details are postponed to Appendix A). Finally, in $\S 6$, we perform several numerical experiments.

\section{Assumptions, Notations, and Standard Results}

We denote by $\mathcal{E}_{h}$ a nondegenerate quasi-uniform rectangular subdivision of the domain $\Omega$ (the quasi-uniformity requirement will only be used in $\S 5$ for establishing the rate of convergence with respect to the polynomials degree). The maximum diameter over all mesh elements is denoted by $h$ and the set of the interior edges is denoted by $\Gamma_{h}$. To each edge $e$ in $\Gamma_{h}$, we associate a unit normal vector $\mathbf{n}_{e}=\left(n_{x}, n_{y}\right)$. We assume that $\mathbf{n}_{e}$ is directed from the element $E^{1}$ to $E^{2}$, where $E^{1}$ denotes a certain element and $E^{2}$ denotes an element that has a common edge with the element $E^{1}$ and a larger index (this simplified element notation will be used throughout the paper). For a boundary edge, $\mathbf{n}_{e}$ is chosen so that it coincides with the outward normal.

The discrete space of discontinuous piecewise polynomials of degree $r$ is denoted by

$$
\mathcal{W}_{r, h}\left(\mathcal{E}_{h}\right)=\left\{w \in L^{2}(\Omega): \forall E \in \mathcal{E}_{h},\left.w\right|_{E} \in \mathrm{P}_{r}(E)\right\}
$$

where $\mathrm{P}_{r}(E)$ is a space of polynomials of degree $r$ over the element $E$. For any function $w \in \mathcal{W}_{r, h}$, we denote the jump and average operators over a given edge $e$ by $[w]$ and $\{w\}$, respectively:

$$
\begin{aligned}
& \text { for an interior edge } e=\partial E^{1} \cap \partial E^{2}, \quad[w]:=w_{e}^{E^{1}}-w_{e}^{E^{2}}, \quad\{w\}:=0.5 w_{e}^{E^{1}}+0.5 w_{e}^{E^{2}}, \\
& \text { for a boundary edge } e=\partial E^{1} \cap \partial \Omega, \quad[w]:=w_{e}^{E^{1}}, \quad\{w\}:=w_{e}^{E^{1}},
\end{aligned}
$$

where $w_{e}^{E^{1}}$ and $w_{e}^{E^{2}}$ are the corresponding polynomial approximations from the elements $E^{1}$ and $E^{2}$. We also recall that the following identity between the jump and the average operators is satisfied:

$$
\left[w_{1} w_{2}\right]=\left\{w_{1}\right\}\left[w_{2}\right]+\left\{w_{2}\right\}\left[w_{1}\right] .
$$

For the finite-element subdivision $\mathcal{E}_{h}$, we define the broken Sobolev space

$$
H^{s}\left(\mathcal{E}_{h}\right)=\left\{w \in L^{2}(\Omega):\left.w\right|_{E^{j}} \in H^{s}\left(E^{j}\right), j=1, \ldots, N_{h}\right\}
$$

with the norms

$$
\|w\|_{0, \Omega}=\left(\sum_{E \in \mathcal{E}_{h}}\|w\|_{0, E}^{2}\right)^{\frac{1}{2}} \text { and }\|w\|_{s, \Omega}=\left(\sum_{E \in \mathcal{E}_{h}}\|w\|_{s, E}^{2}\right)^{\frac{1}{2}}, \quad s>0
$$

where $\|\cdot\|_{s, E}$ denotes the Sobolev $s$-norm over the element $E$.

We now recall some well-known facts that will be used in the error analysis in $§ 5$. First, let us state some approximations properties and inequalities for the finite-element space. 
Lemma 2.1 (hp Approximation, [5, 6]) Let $E \in \mathcal{E}_{h}$ and $\psi \in H^{s}(E), s \geq 0$. Then there exist a positive constant $C$ independent of $\psi, r$, and $h$, and a sequence $\widetilde{\psi}_{r}^{h} \in \mathrm{P}_{r}(E), r=1,2, \ldots$, such that for any $q \in[0, s]$

$$
\left\|\psi-\widetilde{\psi}_{r}^{h}\right\|_{q, E} \leq C \frac{h^{\mu-q}}{r^{s-q}}\|\psi\|_{s, E}, \quad \mu:=\min (r+1, s) .
$$

Lemma 2.2 (Trace Inequalities, [2]) Let $E \in \mathcal{E}_{h}$. Then for the trace operators $\gamma_{0}$ and $\gamma_{1}$, there exists a constant $C_{t}$ independent of $h$ such that

$$
\begin{aligned}
& \forall w \in H^{s}(E), \quad s \geq 1, \quad\left\|\gamma_{0} w\right\|_{0, e} \leq C_{t} h^{-\frac{1}{2}}\left(\|w\|_{0, E}+h\|\nabla w\|_{0, E}\right), \\
& \forall w \in H^{s}(E), \quad s \geq 2, \quad\left\|\gamma_{1} w\right\|_{0, e} \leq C_{t} h^{-\frac{1}{2}}\left(\|\nabla w\|_{0, E}+h\left\|\nabla^{2} w\right\|_{0, E}\right),
\end{aligned}
$$

where $e$ is an edge of the element $E$.

Lemma 2.3 ([39]) Let $E$ be a mesh element with an edge e. Then there is a constant $C_{t}$ independent of $h$ and $r$ such that

$$
\forall w \in \mathrm{P}_{r}(E), \quad\left\|\gamma_{0} w\right\|_{0, e} \leq C_{t} h^{-\frac{1}{2}} r\|w\|_{0, E} .
$$

Lemma $2.4([4,9])$ There exists a constant $C$ independent of $h$ and $r$ such that

$$
\forall w \in \mathcal{W}_{r, h}\left(\mathcal{E}_{h}\right), \quad\|w\|_{0, \Omega}^{2} \leq C\left(\sum_{E \in \mathcal{E}_{h}}\|\nabla w\|_{0, E}^{2}+\sum_{e \in \Gamma_{h}} \frac{1}{|e|}\|[w]\|_{0, e}^{2}\right)^{\frac{1}{2}},
$$

where $|e|$ denotes the measure of $e$.

Lemma 2.5 (Inverse Inequalities) Let $E \in \mathcal{E}_{h}$ and $w \in \mathrm{P}_{r}(E)$. Then there exists a constant $C$ independent of $h$ and $r$ such that

$$
\begin{aligned}
& \|w\|_{L^{\infty}(E)} \leq C h^{-1} r^{2}\|w\|_{0, E}, \\
& \|w\|_{1, E} \leq C h^{-1} r^{2}\|w\|_{0, E} .
\end{aligned}
$$

We also recall the following form of Gronwall's lemma:

Lemma 2.6 (Gronwall) Let $\varphi, \psi$, and $\phi$ be continuous nonnegative functions defined on the interval $a \leq t \leq b$, and the function $\phi$ is nondecreasing. If $\varphi(t)+\psi(t) \leq \phi(t)+\int_{a}^{t} \varphi(s) d s$ for all $t \in[a, b]$, then $\varphi(t)+\psi(t) \leq e^{t-a} \phi(t)$.

In the analysis below we also make the following assumptions:

- $\Omega$ is a rectangular domain with the boundary $\partial \Omega=\partial \Omega_{\text {ver }} \cup \partial \Omega_{\text {hor }}$, where $\partial \Omega_{\text {ver }}$ and $\partial \Omega_{\text {hor }}$ denote the vertical and horizontal pieces of the boundary $\partial \Omega$, respectively. We also split the set if interior edges, $\Gamma_{h}$, into two sets of vertical, $\Gamma_{h}^{\mathrm{ver}}$, and horizontal, $\Gamma_{h}^{\text {hor }}$, edges, respectively;

- The degree of basis polynomials is $r \geq 2$ and the maximum diameter of the elements is $h<1$ (the latter assumption is only needed for simplification of the error analysis). 


\section{Description of the Numerical Scheme}

We consider the Keller-Segel system (1.8). First, notice that the Jacobians of $\mathbf{F}$ and $\mathbf{G}$ are

$$
\frac{\partial \mathbf{F}}{\partial \mathbf{Q}}=\left(\begin{array}{cccc}
\chi u & 0 & \chi \rho & 0 \\
0 & 0 & 0 & 0 \\
0 & -1 & 0 & 0 \\
0 & 0 & 0 & 0
\end{array}\right) \text { and } \frac{\partial \mathbf{G}}{\partial \mathbf{Q}}=\left(\begin{array}{cccc}
\chi v & 0 & 0 & \chi \rho \\
0 & 0 & 0 & 0 \\
0 & 0 & 0 & 0 \\
0 & -1 & 0 & 0
\end{array}\right)
$$

and their eigenvalues are

$$
\lambda_{1}^{\mathbf{F}}=\chi u, \lambda_{2}^{\mathbf{F}}=\lambda_{3}^{\mathbf{F}}=\lambda_{4}^{\mathbf{F}}=0 \quad \text { and } \quad \lambda_{1}^{\mathbf{G}}=\chi v, \quad \lambda_{2}^{\mathbf{G}}=\lambda_{3}^{\mathbf{G}}=\lambda_{4}^{\mathbf{G}}=0,
$$

respectively. Hence, the convective part of (1.8) is hyperbolic. We now design semidiscrete interior penalty Galerkin methods for this system.

We assume that at any time level $t \in[0, T]$ the solution, $(\rho, c, u, v)^{T}$ is approximated by (discontinuous) piecewise polynomials of the corresponding degrees $r_{\rho}, r_{c}, r_{u}$, and $r_{v}$, which satisfy the following relation:

$$
\frac{r_{\max }}{r_{\min }} \leq a, \quad r_{\max }:=\max \left\{r_{\rho}, r_{c}, r_{u}, r_{v}\right\}, r_{\min }:=\min \left\{r_{\rho}, r_{c}, r_{u}, r_{v}\right\}
$$

where $a$ is a constant independent of $r_{\rho}, r_{c}, r_{p}$, and $r_{q}$.

Our new DG methods are formulated as follows. Find a continuous in time solution

$$
\left(\rho^{\mathrm{DG}}(\cdot, t), c^{\mathrm{DG}}(\cdot, t), u^{\mathrm{DG}}(\cdot, t), v^{\mathrm{DG}}(\cdot, t)\right) \in \mathcal{W}_{r_{\rho}, h}^{\rho} \times \mathcal{W}_{r_{c}, h}^{c} \times \mathcal{W}_{r_{u}, h}^{u} \times \mathcal{W}_{r_{v}, h}^{v},
$$

which satisfies the following weak formulation of the chemotaxis system (1.3)-(1.6):

$$
\begin{aligned}
& \int_{\Omega} \rho_{t}^{\mathrm{DG}} w^{\rho}+\sum_{E \in \mathcal{E}_{h}} \int_{E} \nabla \rho^{\mathrm{DG}} \nabla w^{\rho}-\sum_{e \in \Gamma_{h}} \int_{e}\left\{\nabla \rho^{\mathrm{DG}} \cdot \mathbf{n}_{e}\right\}\left[w^{\rho}\right]+\varepsilon \sum_{e \in \Gamma_{h}} \int_{e}\left\{\nabla w^{\rho} \cdot \mathbf{n}_{e}\right\}\left[\rho^{\mathrm{DG}}\right] \\
& \quad+\sigma_{\rho} \sum_{e \in \Gamma_{h}} \frac{r_{\rho}^{2}}{|e|} \int_{e}\left[\rho^{\mathrm{DG}}\right]\left[w^{\rho}\right]-\sum_{E \in \mathcal{E}_{h}} \int_{E} \chi \rho^{\mathrm{DG}} u^{\mathrm{DG}}\left(w^{\rho}\right)_{x}+\sum_{e \in \Gamma_{h}^{\mathrm{ver}}} \int_{e}\left(\chi \rho^{\mathrm{DG}} u^{\mathrm{DG}}\right)^{*} n_{x}\left[w^{\rho}\right] \\
& \quad-\sum_{E \in \mathcal{E}_{h}} \int_{E} \chi \rho^{\mathrm{DG}} v^{\mathrm{DG}}\left(w^{\rho}\right)_{y}+\sum_{e \in \Gamma_{h}^{\mathrm{hor}}} \int_{e}\left(\chi \rho^{\mathrm{DG}} v^{\mathrm{DG}}\right)^{*} n_{y}\left[w^{\rho}\right]=0 \\
& \int_{\Omega} c_{t}^{\mathrm{DG}} w^{c}+\sum_{E \in \mathcal{E}_{h}} \int_{E} \nabla c^{\mathrm{DG}} \nabla w^{c}-\sum_{e \in \Gamma_{h}} \int_{e}\left\{\nabla c^{\mathrm{DG}} \cdot \mathbf{n}_{e}\right\}\left[w^{c}\right]+\varepsilon \sum_{e \in \Gamma_{h}} \int_{e}\left\{\nabla w^{c} \cdot \mathbf{n}_{e}\right\}\left[c^{\mathrm{DG}}\right] \\
& \quad+\sigma_{c} \sum_{e \in \Gamma_{h}} \frac{r_{c}^{2}}{|e|} \int_{e}\left[c^{\mathrm{DG}}\right]\left[w^{c}\right]+\int_{\Omega} c^{\mathrm{DG}} w^{c}-\int_{\Omega} \rho^{\mathrm{DG}} w^{c}=0 \\
& \int_{\Omega} u^{\mathrm{DG}} w^{u}+\sum_{E \in \mathcal{E}_{h}} \int_{E} c^{\mathrm{DG}}\left(w^{u}\right)_{x}+\sum_{e \in \Gamma_{h}^{\mathrm{ver}}} \int_{e}\left(-c^{\mathrm{DG}}\right)_{u}^{*} n_{x}\left[w^{u}\right] \\
& \quad-\sum_{e \in \partial \Omega_{\mathrm{ver}}} \int_{e} c^{\mathrm{DG}} n_{x} w^{u}+\sigma_{u} \sum_{e \in \Gamma_{h} \cup \partial \Omega_{\mathrm{ver}}} \frac{r_{u}^{2}}{|e|} \int_{e}\left[u^{\mathrm{DG}}\right]\left[w^{u}\right]=0
\end{aligned}
$$




$$
\begin{aligned}
& \int_{\Omega} v^{\mathrm{DG}} w^{v}+\sum_{E \in \mathcal{E}_{h}} \int_{E} c^{\mathrm{DG}}\left(w^{v}\right)_{y}+\sum_{e \in \Gamma_{h}^{\text {hor }}} \int_{e}\left(-c^{\mathrm{DG}}\right)_{v}^{*} n_{y}\left[w^{v}\right] \\
& -\sum_{e \in \partial \Omega_{\mathrm{hor}}} \int_{e} c^{\mathrm{DG}} n_{y} w^{v}+\sigma_{v} \sum_{e \in \Gamma_{h} \cup \partial \Omega_{\mathrm{hor}}} \frac{r_{v}^{2}}{|e|} \int_{e}\left[v^{\mathrm{DG}}\right]\left[w^{v}\right]=0,
\end{aligned}
$$

and the initial conditions:

$$
\begin{aligned}
& \int_{\Omega} \rho^{\mathrm{DG}}(\cdot, 0) w^{\rho}=\int_{\Omega} \rho(\cdot, 0) w^{\rho}, \quad \int_{\Omega} c^{\mathrm{DG}}(\cdot, 0) w^{c}=\int_{\Omega} c(\cdot, 0) w^{c}, \\
& \int_{\Omega}^{\Omega} u^{\mathrm{DG}}(\cdot, 0) w^{u}=\int_{\Omega}^{\Omega} u(\cdot, 0) w^{u}, \quad \int_{\Omega} v^{\mathrm{DG}}(\cdot, 0) w^{v}=\int_{\Omega} v(\cdot, 0) w^{v} .
\end{aligned}
$$

Here, $\left(w^{\rho}, w^{c}, w^{u}, w^{v}\right) \in \mathcal{W}_{r_{\rho}, h}^{\rho} \times \mathcal{W}_{r_{c}, h}^{c} \times \mathcal{W}_{r_{u}, h}^{u} \times \mathcal{W}_{r_{v}, h}^{v}$ are the test functions, $\sigma_{\rho}, \sigma_{c}, \sigma_{u}$ and $\sigma_{v}$ are real positive penalty parameters. The parameter $\varepsilon$ is equal to either $-1,0$, or 1 : these values of $\varepsilon$ correspond to the SIPG, IIPG, or NIPG method, respectively.

To approximate the convective terms in (3.3) and (3.5)-(3.6), we use the central-upwind fluxes from [32]:

$$
\begin{aligned}
& \left(\chi \rho^{\mathrm{DG}} u^{\mathrm{DG}}\right)^{*}=\frac{a^{\text {out }}\left(\chi \rho^{\mathrm{DG}} u^{\mathrm{DG}}\right)_{e}^{E^{1}}-a^{\text {in }}\left(\chi \rho^{\mathrm{DG}} u^{\mathrm{DG}}\right)_{e}^{E^{2}}}{a^{\text {out }}-a^{\text {in }}}-\frac{a^{\text {out }} a^{\text {in }}}{a^{\text {out }}-a^{\text {in }}}\left[\rho^{\mathrm{DG}}\right], \\
& \left(\chi \rho^{\mathrm{DG}} v^{\mathrm{DG}}\right)^{*}=\frac{b^{\text {out }}\left(\chi \rho^{\mathrm{DG}} v^{\mathrm{DG}}\right)_{e}^{E^{1}}-b^{\text {in }}\left(\chi \rho^{\mathrm{DG}} v^{\mathrm{DG}}\right)_{e}^{E^{2}}}{b^{\text {out }}-b^{\text {in }}}-\frac{b^{\text {out }} b^{\text {in }}}{b^{\text {out }}-b^{\text {in }}}\left[\rho^{\mathrm{DG}}\right], \\
& \left(-c^{\mathrm{DG}}\right)_{u}^{*}=-\frac{a^{\text {out }}\left(c^{\mathrm{DG}}\right)_{e}^{E^{1}}-a^{\text {in }}\left(c^{\mathrm{DG}}\right)_{e}^{E^{2}}}{a^{\text {out }}-a^{\text {in }}}-\frac{a^{\text {out }} a^{\text {in }}}{a^{\text {out }}-a^{\text {in }}}\left[u^{\mathrm{DG}}\right], \\
& \left(-c^{\mathrm{DG}}\right)_{v}^{*}=-\frac{b^{\text {out }}\left(c^{\mathrm{DG}}\right)_{e}^{E^{1}}-b^{\text {in }}\left(c^{\mathrm{DG}}\right)_{e}^{E^{2}}}{b^{\text {out }}-b^{\text {in }}}-\frac{b^{\text {out }} b^{\text {in }}}{b^{\text {out }}-b^{\text {in }}}\left[v^{\mathrm{DG}}\right] .
\end{aligned}
$$

Here, $a^{\text {out }}, a^{\text {in }}, b^{\text {out }}$, and $b^{\text {in }}$ are the one-sided local speeds in the $x$ - and $y$-directions. Since the convective part of the system (1.3)-(1.6) is hyperbolic, these speeds can be estimated using the largest and the smallest eigenvalues of the Jacobian $\frac{\partial \mathbf{F}}{\partial \mathbf{Q}}$ and $\frac{\partial \mathbf{G}}{\partial \mathbf{Q}}($ see $(3.1))$ :

$$
\begin{aligned}
& a^{\text {out }}=\max \left(\left(\chi u^{\mathrm{DG}}\right)_{e}^{E^{1}},\left(\chi u^{\mathrm{DG}}\right)_{e}^{E^{2}}, 0\right), \quad a^{\text {in }}=\min \left(\left(\chi u^{\mathrm{DG}}\right)_{e}^{E^{1}},\left(\chi u^{\mathrm{DG}}\right)_{e}^{E^{2}}, 0\right), \\
& b^{\text {out }}=\max \left(\left(\chi v^{\mathrm{DG}}\right)_{e}^{E^{1}},\left(\chi v^{\mathrm{DG}}\right)_{e}^{E^{2}}, 0\right), \quad b^{\text {in }}=\min \left(\left(\chi v^{\mathrm{DG}}\right)_{e}^{E^{1}},\left(\chi v^{\mathrm{DG}}\right)_{e}^{E^{2}}, 0\right) .
\end{aligned}
$$

Remark. If $a^{\text {out }}-a^{\text {in }}=0$ at a certain element edge $e$, we set

$$
\begin{aligned}
& \left(\chi \rho^{\mathrm{DG}} u^{\mathrm{DG}}\right)^{*}=\frac{\left(\chi \rho^{\mathrm{DG}} u^{\mathrm{DG}}\right)_{e}^{E^{1}}+\left(\chi \rho^{\mathrm{DG}} u^{\mathrm{DG}}\right)_{e}^{E^{2}}}{2}, \quad\left(\chi \rho^{\mathrm{DG}} v^{\mathrm{DG}}\right)^{*}=\frac{\left(\chi \rho^{\mathrm{DG}} v^{\mathrm{DG}}\right)_{e}^{E^{1}}+\left(\chi \rho^{\mathrm{DG}} v^{\mathrm{DG}}\right)_{e}^{E^{2}}}{2}, \\
& \left(-c^{\mathrm{DG}}\right)_{u}^{*}=-\frac{\left(c^{\mathrm{DG}}\right)_{e}^{E^{1}}+\left(c^{\mathrm{DG}}\right)_{e}^{E^{2}}}{2}, \quad\left(-c^{\mathrm{DG}}\right)_{v}^{*}=-\frac{\left(c^{\mathrm{DG}}\right)_{e}^{E^{1}}+\left(c^{\mathrm{DG}}\right)_{e}^{E^{2}}}{2}
\end{aligned}
$$

there. Notice that in any case, the following inequalities,

$$
\frac{a^{\text {out }}}{a^{\text {out }}-a^{\text {in }}} \leq 1, \quad \frac{-a^{\text {in }}}{a^{\text {out }}-a^{\text {in }}} \leq 1, \quad \frac{b^{\text {out }}}{b^{\text {out }}-b^{\text {in }}} \leq 1, \quad \text { and } \quad \frac{-b^{\text {in }}}{b^{\text {out }}-b^{\text {in }}} \leq 1,
$$


are satisfied.

From now on we will assume that $a^{\text {out }}-a^{\text {in }}>0$ and $b^{\text {out }}-b^{\text {in }}>0$ throughout the computational domain.

\section{Consistency of the Numerical Scheme}

In this section, we show that the proposed DG methods (3.3)-(3.6) are strongly consistent with the Keller-Segel system (1.3)-(1.6).

Lemma 4.1 If the solution of (1.3)-(1.6) is sufficiently regular, namely, if $(\rho, c) \in H^{1}([0, T]) \cap$ $H^{2}\left(\mathcal{E}_{h}\right)$ and $(u, v) \in L^{2}([0, T]) \cap H^{2}\left(\mathcal{E}_{h}\right)$, then it satisfies the formulation (3.3)-(3.6).

Proof: We first multiply equation $(1.3)$ by $w^{\rho} \in \mathcal{W}_{r_{\rho}, h}^{\rho}$ and integrate by parts on one element $E$ to obtain

$\int_{E} \rho_{t} w^{\rho}+\int_{E} \nabla \rho \nabla w^{\rho}-\int_{\partial E} \nabla \rho \cdot \mathbf{n}_{e} w^{\rho}-\int_{E} \chi \rho u\left(w^{\rho}\right)_{x}+\int_{\partial E} \chi \rho u n_{x} w^{\rho}-\int_{E} \chi \rho v\left(w^{\rho}\right)_{y}+\int_{\partial E} \chi \rho v n_{y} w^{\rho}=0$.

Notice that continuity of $\rho$ and $u$ implies that at the edge $e, \rho_{e}^{E^{1}}=\rho_{e}^{E^{2}}$ and $(\chi \rho u)_{e}^{E^{1}}=(\chi \rho u)_{e}^{E^{2}}$. Therefore, $[\rho]=0$ and

$$
\begin{aligned}
\{\chi \rho u\} & =\frac{1}{2}(\chi \rho u)_{e}^{E^{1}}+\frac{1}{2}(\chi \rho u)_{e}^{E^{2}}=(\chi \rho u)_{e}^{E^{1}}=\frac{a^{\text {out }}-a^{\text {in }}}{a^{\text {out }}-a^{\text {in }}}(\chi \rho u)_{e}^{E^{1}} \\
& =\frac{a^{\text {out }}}{a^{\text {out }}-a^{\text {in }}}(\chi \rho u)_{e}^{E^{1}}-\frac{a^{\text {in }}}{a^{\text {out }}-a^{\text {in }}}(\chi \rho u)_{e}^{E^{2}}=\frac{a^{\text {out }}(\chi \rho u)_{e}^{E^{1}}-a^{\text {in }}(\chi \rho u)_{e}^{E^{2}}}{a^{\text {out }}-a^{\text {in }}}=(\chi \rho u)^{*} .
\end{aligned}
$$

Summing now equation (4.1) over all elements $E \in \mathcal{E}_{h}$, using the jump-average identity (2.1), adding the penalty terms $\varepsilon \sum_{e \in \Gamma_{h}} \int_{e}\left\{\nabla w^{\rho} \cdot \mathbf{n}_{e}\right\}[\rho]$ and $\sigma_{\rho} \sum_{e \in \Gamma_{h}} \frac{r_{\rho}^{2}}{|e|} \int_{e}[\rho]\left[w^{\rho}\right]$, and using the Neumann boundary conditions (1.7), we obtain that the solution of the system (1.3)-(1.6) satisfies equation (3.3). A similar procedure can be applied to show that the solution of (1.3)-(1.6) satisfies equations (3.4)-(3.6) as well. This concludes the consistency proof.

\section{Error Analysis}

In this section, we prove the existence and show the convergence of the numerical solution using the Schauder's fixed point theorem, [24].

In the analysis below we will assume that the exact solution of the system (1.3)-(1.6) is sufficiently regular for $t \leq T$, where $T$ is a pre-blow-up time. In particular we will assume that

$$
(\rho, c, u, v) \in H^{s_{1}}([0, T]) \cap H^{s_{2}}(\Omega), \quad s_{1}>3 / 2, \quad s_{2} \geq 3,
$$

which is needed for the $h$-analysis (convergence rate with respect to the mesh size), or

$$
(\rho, c, u, v) \in H^{s_{1}}([0, T]) \cap H^{s_{2}}(\Omega), \quad s_{1}>3 / 2, \quad s_{2} \geq 6,
$$


which is needed for the $r$-analysis (convergence rate with respect to the polynomial degree). Notice that these assumptions are reasonable since classical solutions of the Keller-Segel system (1.1) are regular (before the blow-up time) provided the initial data are sufficiently smooth, see [26] and references therein.

We denote by $\widetilde{\rho}, \widetilde{c}, \widetilde{u}$, and $\widetilde{v}$ the piecewise polynomial interpolants of the exact solution components $\rho, c, u$, and $v$ of the Keller-Segel system (1.3)-(1.6) and assume that these interpolants satisfy the approximation property (2.2). We then use the idea similar to [36] and define the following subset of the broken Sobolev space:

$$
\begin{aligned}
& S=\left\{\left(\phi^{\rho}, \phi^{c}, \phi^{u}, \phi^{v}\right) \in L^{2}([0, T]) \cap L^{\infty}([0, T]) \cap \mathcal{W}_{r_{\rho}, h}^{\rho} \times \mathcal{W}_{r_{c}, h}^{c} \times \mathcal{W}_{r_{u}, h}^{u} \times \mathcal{W}_{r_{v}, h}^{v}:\right. \\
& \sup _{t \in[0, T]}\left\|\phi^{\rho}-\widetilde{\rho}\right\|_{0, \Omega}^{2}+\int_{0}^{T}\left(\left\|\mid \nabla\left(\phi^{\rho}-\widetilde{\rho}\right)\right\|\left\|_{0, \Omega}^{2}+\sum_{e \in \Gamma_{h}} \frac{r_{\rho}^{2}}{|e|}\right\|\left[\left(\phi^{\rho}-\widetilde{\rho}\right)\right] \|_{0, e}^{2}\right) \\
& \leq C_{\rho}\left(\frac{h^{2 \min \left(r_{\rho}+1, s_{\rho}\right)-2}}{r_{\rho}^{2 s_{\rho}-4}}+\frac{h^{2 \min \left(r_{c}+1, s_{c}\right)-2}}{r_{c}^{2 s_{c}-4}}+\frac{h^{2 \min \left(r_{u}+1, s_{u}\right)-2}}{r_{u}^{2 s_{u}-4}}+\frac{h^{2 \min \left(r_{v}+1, s_{v}\right)-2}}{r_{v}^{2 s_{v}-4}}\right), \\
& \sup _{t \in[0, T]}\left\|\phi^{c}-\widetilde{c}\right\|_{0, \Omega}^{2}+\int_{0}^{T}\left(\left.\left\|\nabla \nabla\left(\phi^{c}-\widetilde{c}\right)\right\|\right|_{0, \Omega} ^{2}+\sum_{e \in \Gamma_{h}} \frac{r_{c}^{2}}{|e|}\left\|\left[\left(\phi^{c}-\widetilde{c}\right)\right]\right\|_{0, e}^{2}\right) \\
& \leq C_{c}\left(\frac{h^{2 \min \left(r_{\rho}+1, s_{\rho}\right)-2}}{r_{\rho}^{2 s_{\rho}-4}}+\frac{h^{2 \min \left(r_{c}+1, s_{c}\right)-2}}{r_{c}^{2 s_{c}-4}}+\frac{h^{2 \min \left(r_{u}+1, s_{u}\right)-2}}{r_{u}^{2 s_{u}-4}}+\frac{h^{2 \min \left(r_{v}+1, s_{v}\right)-2}}{r_{v}^{2 s_{v}-4}}\right), \\
& \sup _{[0, T]}\left\|\phi^{u}-\widetilde{u}\right\|_{0, \Omega} \leq C h\left(\frac{1}{r_{\rho}^{2}}+\frac{1}{r_{c}^{2}}+\frac{1}{r_{u}^{2}}+\frac{1}{r_{v}^{2}}\right) \text {, } \\
& \int_{0}^{T}\left(\left\|\phi^{u}-\widetilde{u}\right\|_{0, \Omega}^{2}+\sum_{e \in \Gamma_{h}} \frac{r_{u}^{2}}{|e|}\left\|\left[\left(\phi^{u}-\widetilde{u}\right)\right]\right\|_{0, e}^{2}\right) \\
& \leq C_{u}\left(\frac{h^{2 \min \left(r_{\rho}+1, s_{\rho}\right)-2}}{r_{\rho}^{2 s_{\rho}-4}}+\frac{h^{2 \min \left(r_{c}+1, s_{c}\right)-2}}{r_{c}^{2 s_{c}-4}}+\frac{h^{2 \min \left(r_{u}+1, s_{u}\right)-2}}{r_{u}^{2 s_{u}-4}}+\frac{h^{2 \min \left(r_{v}+1, s_{v}\right)-2}}{r_{v}^{2 s_{v}-4}}\right), \\
& \sup _{[0, T]}\left\|\phi^{v}-\widetilde{v}\right\|_{0, \Omega} \leq C h\left(\frac{1}{r_{\rho}^{2}}+\frac{1}{r_{c}^{2}}+\frac{1}{r_{u}^{2}}+\frac{1}{r_{v}^{2}}\right), \\
& \int_{0}^{T}\left(\left\|\phi^{v}-\widetilde{v}\right\|_{0, \Omega}^{2}+\sum_{e \in \Gamma_{h}} \frac{r_{v}^{2}}{|e|}\left\|\left[\left(\phi^{v}-\widetilde{v}\right)\right]\right\|_{0, e}^{2}\right) \\
& \left.\leq C_{v}\left(\frac{h^{2 \min \left(r_{\rho}+1, s_{\rho}\right)-2}}{r_{\rho}^{2 s_{\rho}-4}}+\frac{h^{2 \min \left(r_{c}+1, s_{c}\right)-2}}{r_{c}^{2 s_{c}-4}}+\frac{h^{2 \min \left(r_{u}+1, s_{u}\right)-2}}{r_{u}^{2 s_{u}-4}}+\frac{h^{2 \min \left(r_{v}+1, s_{v}\right)-2}}{r_{v}^{2 s_{v}-4}}\right)\right\},
\end{aligned}
$$

where $C, C_{\rho}, C_{c}, C_{u}$, and $C_{v}$ are positive constants (which will be defined later) independent of $h$ and the polynomial degrees $\left(r_{\rho}, r_{c}, r_{u}, r_{v}\right)$, and the parameters $s_{\rho}, s_{c}, s_{u}$, and $s_{v}$ denote the regularity of the corresponding components of the exact solution. Clearly the subset $S$ is a closed convex subset of the broken Sobolev space and it is not empty since it contains the element $(\widetilde{\rho}, \widetilde{c}, \widetilde{u}, \widetilde{v})$. We first show that the functions in $S$ are bounded. 
Lemma 5.1 For any $\left(\phi^{\rho}, \phi^{c}, \phi^{u}, \phi^{v}\right) \in S$, there exist positive constants $M_{\rho}, M_{c}, M_{u}$, and $M_{v}$ independent of $h, r_{\rho}, r_{c}, r_{u}$, and $r_{v}$, such that

$$
\sup _{t \in[0, T]}\left\|\phi^{\rho}\right\|_{\infty, \Omega} \leq M_{\rho}, \quad \sup _{t \in[0, T]}\left\|\phi^{c}\right\|_{\infty, \Omega} \leq M_{c}, \quad \sup _{t \in[0, T]}\left\|\phi^{u}\right\|_{\infty, \Omega} \leq M_{u}, \quad \sup _{t \in[0, T]}\left\|\phi^{v}\right\|_{\infty, \Omega} \leq M_{v} .
$$

Proof: From the definition of the subset $S$, we have

$\sup _{t \in[0, T]}\left\|\phi^{\rho}-\widetilde{\rho}\right\|_{0, \Omega}^{2} \leq C_{\rho}\left(\frac{h^{2 \min \left(r_{\rho}+1, s_{\rho}\right)-2}}{r_{\rho}^{2 s_{\rho}-4}}+\frac{h^{2 \min \left(r_{c}+1, s_{c}\right)-2}}{r_{c}^{2 s_{c}-4}}+\frac{h^{2 \min \left(r_{u}+1, s_{u}\right)-2}}{r_{u}^{2 s_{u}-4}}+\frac{h^{2 \min \left(r_{v}+1, s_{v}\right)-2}}{r_{v}^{2 s_{v}-4}}\right)$.

Hence,

$$
\sup _{t \in[0, T]}\left\|\phi^{\rho}-\widetilde{\rho}\right\|_{0, \Omega} \leq M \frac{h}{r_{\min }^{2}}
$$

Using the inverse inequality (2.6), we obtain

$$
\sup _{t \in[0, T]}\left\|\phi^{\rho}-\widetilde{\rho}\right\|_{\infty, \Omega} \leq M_{1} r_{\rho}^{2} h^{-1} \sup _{t \in[0, T]}\left\|\phi^{\rho}-\widetilde{\rho}\right\|_{0, \Omega} \leq \frac{r_{\max }}{r_{\min }} M^{*} \leq M .
$$

This estimate implies that

$$
\sup _{t \in[0, T]}\left\|\phi^{\rho}\right\|_{\infty, \Omega} \leq M+\sup _{[0, T]}\|\widetilde{\rho}\|_{\infty, \Omega}
$$

which, together with the $h p$ approximation property (see Lemma 2.1), yields the first bound in (5.3). The remaining three estimates in (5.3) are obtained in a similar manner.

We now define the solution operator $A$ on $S$ as follows:

$$
\forall\left(\phi^{\rho}, \phi^{c}, \phi^{u}, \phi^{v}\right) \in S, \quad A\left(\phi^{\rho}, \phi^{c}, \phi^{u}, \phi^{v}\right)=\left(\phi_{L}^{\rho}, \phi_{L}^{c}, \phi_{L}^{u}, \phi_{L}^{v}\right),
$$

where the initial conditions are $\left(\phi_{L}^{\rho, 0}, \phi_{L}^{c, 0}, \phi_{L}^{u, 0}, \phi_{L}^{v, 0}\right)=\left(\widetilde{\rho}^{0}, \widetilde{c}^{0}, \widetilde{u}^{0}, \widetilde{v}^{0}\right)$, and the functions

$$
\begin{aligned}
& \phi_{L}^{\rho} \in \mathcal{W}_{r_{\rho}, h, t}^{\rho}:=H^{s}([0, T]) \cap \mathcal{W}_{r_{\rho}, h}^{\rho}, \quad \phi_{L}^{c} \in \mathcal{W}_{r_{c}, h, t}^{c}:=H^{s}([0, T]) \cap \mathcal{W}_{r_{c}, h}^{c}, \quad s>3 / 2 \\
& \phi_{L}^{u} \in \mathcal{W}_{r_{u}, h, t}^{u}:=L^{2}([0, T]) \cap L^{\infty}([0, T]) \cap \mathcal{W}_{r_{u}, h}^{u}, \quad \phi_{L}^{v} \in \mathcal{W}_{r_{v}, h, t}^{v}:=L^{2}([0, T]) \cap L^{\infty}([0, T]) \cap \mathcal{W}_{r_{v}, h}^{v}
\end{aligned}
$$

are such that

$$
\begin{aligned}
\int_{\Omega}\left(\phi_{L}^{\rho}\right)_{t} w^{\rho} & +\sum_{E \in \mathcal{E}_{h}} \int_{E} \nabla\left(\phi_{L}^{\rho}\right) \nabla w^{\rho}-\sum_{e \in \Gamma_{h}} \int_{e}\left\{\nabla \phi_{L}^{\rho} \cdot \mathbf{n}_{e}\right\}\left[w^{\rho}\right]+\varepsilon \sum_{e \in \Gamma_{h}} \int_{e}\left\{\nabla w^{\rho} \cdot \mathbf{n}_{e}\right\}\left[\phi_{L}^{\rho}\right] \\
& +\sigma_{\rho} \sum_{e \in \Gamma_{h}} \frac{r_{\rho}^{2}}{|e|} \int_{e}\left[\phi_{L}^{\rho}\right]\left[w^{\rho}\right]-\sum_{E \in \mathcal{E}_{h}} \int_{E} \chi \phi_{L}^{\rho} \phi^{u}\left(w^{\rho}\right)_{x}+\sum_{e \in \Gamma_{h}^{\mathrm{ver}}} \int_{e}\left(\chi \phi_{L}^{\rho} \phi^{u}\right)^{*} n_{x}\left[w^{\rho}\right] \\
& -\sum_{E \in \mathcal{E}_{h}} \int_{E} \chi \phi_{L}^{\rho} \phi^{v}\left(w^{\rho}\right)_{y}+\sum_{e \in \Gamma_{h}^{\mathrm{hor}}} \int_{e}\left(\chi \phi_{L}^{\rho} \phi^{v}\right)^{*} n_{y}\left[w^{\rho}\right]=0, \quad \forall w^{\rho} \in \mathcal{W}_{r_{\rho}, h}^{\rho}, \\
\int_{\Omega}\left(\phi_{L}^{c}\right)_{t} w^{c} & +\sum_{E \in \mathcal{E}_{h}} \int_{E} \nabla \phi_{L}^{c} \nabla w^{c}-\sum_{e \in \Gamma_{h}} \int_{e}\left\{\nabla \phi_{L}^{c} \cdot \mathbf{n}_{e}\right\}\left[w^{c}\right]+\varepsilon \sum_{e \in \Gamma_{h}} \int_{e}\left\{\nabla w^{c} \cdot \mathbf{n}_{e}\right\}\left[\phi_{L}^{c}\right] \\
& +\sigma_{c} \sum_{e \in \Gamma_{h}} \frac{r_{c}^{2}}{|e|} \int_{e}\left[\phi_{L}^{c}\right]\left[w^{c}\right]+\int_{\Omega} \phi_{L}^{c} w^{c}-\int_{\Omega} \phi_{L}^{\rho} w^{c}=0, \quad \forall w^{c} \in \mathcal{W}_{r_{c}, h}^{c},
\end{aligned}
$$




$$
\begin{aligned}
\int_{\Omega} \phi_{L}^{u} w^{u} & +\sum_{E \in \mathcal{E}_{h}} \int_{E} \phi_{L}^{c}\left(w^{u}\right)_{x}+\sum_{e \in \Gamma_{h}^{\mathrm{ver}}} \int_{e}\left(-\phi_{L}^{c}\right)_{u}^{*} n_{x}\left[w^{u}\right]-\sum_{e \in \partial \Omega_{\mathrm{ver}}} \int_{e} \phi_{L}^{c} n_{x} w^{u} \\
& +\sigma_{u} \sum_{e \in \Gamma_{h} \cup \partial \Omega_{\mathrm{ver}}} \frac{r_{u}^{2}}{|e|} \int_{e}\left[\phi_{L}^{u}\right]\left[w^{u}\right]=0, \quad \forall w^{u} \in \mathcal{W}_{r_{u}, h}^{u} \\
\int_{\Omega} \phi_{L}^{v} w^{v} & +\sum_{E \in \mathcal{E}_{h}} \int_{E} \phi_{L}^{c}\left(w^{v}\right)_{y}+\sum_{e \in \Gamma_{h}^{\mathrm{hor}}} \int_{e}\left(-\phi_{L}^{c}\right)_{v}^{*} n_{y}\left[w^{v}\right]-\sum_{e \in \partial \Omega_{\mathrm{hor}}} \int_{e} \phi_{L}^{c} n_{y} w^{v} \\
& +\sigma_{v} \sum_{e \in \Gamma_{h} \cup \partial \Omega_{\mathrm{hor}}} \frac{r_{v}^{2}}{|e|} \int_{e}\left[\phi_{L}^{v}\right]\left[w^{v}\right]=0, \quad \forall w^{v} \in \mathcal{W}_{r_{v}, h}^{v} .
\end{aligned}
$$

As before, the central-upwind numerical fluxes are utilized in (5.4)-(5.7):

$$
\begin{aligned}
& \left(\chi \phi_{L}^{\rho} \phi^{u}\right)^{*}=\frac{a_{L}^{\text {out }}\left(\chi \phi_{L}^{\rho} \phi^{u}\right)_{e}^{E^{1}}-a_{L}^{\text {in }}\left(\chi \phi_{L}^{\rho} \phi^{u}\right)_{e}^{E^{2}}}{a_{L}^{\text {out }}-a_{L}^{\text {in }}}-\frac{a_{L}^{\text {out }} a_{L}^{\text {in }}}{a_{L}^{\text {out }}-a_{L}^{\text {in }}}\left[\phi_{L}^{\rho}\right], \\
& \left(\chi \phi_{L}^{\rho} \phi^{v}\right)^{*}=\frac{b_{L}^{\text {out }}\left(\chi \phi_{L}^{\rho} \phi^{v}\right)_{e}^{E^{1}}-b_{L}^{\text {in }}\left(\chi \phi_{L}^{\rho} \phi^{v}\right)_{e}^{E^{2}}}{b_{L}^{\text {out }}-b_{L}^{\text {in }}}-\frac{b_{L}^{\text {out }} b_{L}^{\text {in }}}{b_{L}^{\text {out }}-b_{L}^{\text {in }}}\left[\phi_{L}^{\rho}\right], \\
& \left(-\phi_{L}^{c}\right)_{u}^{*}=-\frac{a_{L}^{\text {out }}\left(\phi_{L}^{c}\right)_{e}^{E^{1}}-a_{L}^{\text {in }}\left(\phi_{L}^{c}\right)_{e}^{E^{2}}}{a_{L}^{\text {out }}-a_{L}^{\text {in }}}-\frac{a_{L}^{\text {out }} a_{L}^{\text {in }}}{a_{L}^{\text {out }}-a_{L}^{\text {in }}}\left[\phi_{L}^{u}\right], \\
& \left(-\phi_{L}^{c}\right)_{v}^{*}=-\frac{b_{L}^{\text {out }}\left(\phi_{L}^{c}\right)_{e}^{E^{1}}-b_{L}^{\text {in }}\left(\phi_{L}^{c}\right)_{e}^{E^{2}}}{b_{L}^{\text {out }}-b_{L}^{\text {in }}}-\frac{b_{L}^{\text {out }} b_{L}^{\text {in }}}{b_{L}^{\text {out }}-b_{L}^{\text {in }}}\left[\phi_{L}^{v}\right]
\end{aligned}
$$

where the one-sided local speeds are:

$$
\begin{aligned}
& a_{L}^{\text {out }}:=\max \left(\left(\chi \phi^{u}\right)_{e}^{E^{1}},\left(\chi \phi^{u}\right)_{e}^{E^{2}}, 0\right), \quad a_{L}^{\text {in }}:=\min \left(\left(\chi \phi^{u}\right)_{e}^{E^{1}},\left(\chi \phi^{u}\right)_{e}^{E^{2}}, 0\right), \\
& b_{L}^{\text {out }}:=\max \left(\left(\chi \phi^{v}\right)_{e}^{E^{1}},\left(\chi \phi^{v}\right)_{e}^{E^{2}}, 0\right), \quad b_{L}^{\text {in }}:=\min \left(\left(\chi \phi^{v}\right)_{e}^{E^{1}},\left(\chi \phi^{v}\right)_{e}^{E^{2}}, 0\right) .
\end{aligned}
$$

Notice that the inequalities similar to (3.10),

$$
\frac{a_{L}^{\text {out }}}{a_{L}^{\text {out }}-a_{L}^{\text {in }}} \leq 1, \quad \frac{-a_{L}^{\text {in }}}{a_{L}^{\text {out }}-a_{L}^{\text {in }}} \leq 1, \quad \frac{b_{L}^{\text {out }}}{b_{L}^{\text {out }}-b_{L}^{\text {in }}} \leq 1, \quad \text { and } \quad \frac{-b_{L}^{\text {in }}}{b_{L}^{\text {out }}-b_{L}^{\text {in }}} \leq 1,
$$

which are needed in our convergence proof, are satisfied for the local speeds defined in (5.9) as well (for simplicity, we assume that $a^{\text {out }}-a^{\text {in }} \neq 0$ and $b^{\text {out }}-b^{\text {in }} \neq 0$ throughout the computational domain).

We now show that the operator $A$ is well-defined by proving existence and uniqueness of $\left(\phi_{L}^{\rho}, \phi_{L}^{c}, \phi_{L}^{u}, \phi_{L}^{v}\right)$.

Lemma 5.2 There exists a unique solution $\left(\phi_{L}^{\rho}, \phi_{L}^{c}, \phi_{L}^{u}, \phi_{L}^{v}\right) \in \mathcal{W}_{r_{\rho}, h, t}^{\rho} \times \mathcal{W}_{r_{c}, h, t}^{c} \times \mathcal{W}_{r_{u}, h, t}^{u} \times \mathcal{W}_{r_{v}, h, t}^{v}$ of (5.4)-(5.7).

Proof: First, notice that equations (5.4)-(5.5) can be rewritten as the explicit linear differential equations for $\phi_{L}^{\rho}$ and $\phi_{L}^{c}$. Hence, there exists a unique solution $\left(\phi_{L}^{\rho}, \phi_{L}^{c}\right) \in \mathcal{W}_{r_{\rho}, h, t}^{\rho} \times \mathcal{W}_{r_{c}, h, t}^{c}$. 
Equations (5.6)-(5.7) can be rewritten as

$$
\begin{aligned}
\int_{\Omega} \phi_{L}^{u} w^{u}+\sigma_{u} \sum_{e \in \Gamma_{h} \cup \partial \Omega_{\mathrm{ver}}} \frac{r_{u}^{2}}{|e|} \int_{e}\left[\phi_{L}^{u}\right]\left[w^{u}\right] & =-\sum_{E \in \mathcal{E}_{h}} \int_{E} \phi_{L}^{c}\left(w^{u}\right)_{x}-\sum_{e \in \Gamma_{h}^{\mathrm{ver}}} \int_{e}\left(-\phi_{L}^{c}\right)_{u}^{*} n_{x}\left[w^{u}\right] \\
& +\sum_{e \in \partial \Omega_{\mathrm{ver}}} \int_{e} \phi_{L}^{c} n_{x} w^{u}, \quad \forall w^{u} \in \mathcal{W}_{r_{u}, h}^{u} \\
\int_{\Omega} \phi_{L}^{v} w^{v}+\sigma_{v} \sum_{e \in \Gamma_{h} \cup \partial \Omega_{\mathrm{hor}}} \frac{r_{v}^{2}}{|e|} \int_{e}\left[\phi_{L}^{v}\right]\left[w^{v}\right] & =-\sum_{E \in \mathcal{E}_{h}} \int_{E} \phi_{L}^{c}\left(w^{v}\right)_{y}-\sum_{e \in \Gamma_{h}^{\mathrm{hor}}} \int_{e}\left(-\phi_{L}^{c}\right)_{v}^{*} n_{y}\left[w^{v}\right] \\
& +\sum_{e \in \partial \Omega_{\mathrm{hor}}} \int_{e} \phi_{L}^{c} n_{y} w^{v}, \quad \forall w^{v} \in \mathcal{W}_{r_{v}, h}^{v} .
\end{aligned}
$$

The bilinear form on the left-hand side (LHS) of equation (5.11) is coercive since for all $\varphi \in \mathcal{W}_{r_{u}, h}^{u}$,

$$
\int_{\Omega} \varphi \varphi+\sigma_{u} \sum_{e \in \Gamma_{h} \cup \partial \Omega_{\mathrm{ver}}} \frac{r_{u}^{2}}{|e|} \int_{e}[\varphi][\varphi] \geq\|\varphi\|_{0, \Omega}^{2} .
$$

It is also continuous on $\mathcal{W}_{r_{u}, h}^{u} \times \mathcal{W}_{r_{u}, h}^{u}$, while the linear form on the right-hand side (RHS) of (5.11) is continuous on $\mathcal{W}_{r_{u}, h}^{u}$. Hence, there exists a unique solution of (5.11). The same argument is true for equation (5.12). This concludes the proof of the lemma.

Our next goal is to show that the operator $A$ maps $S$ into itself and that $A$ is compact. By the second Shauder fixed-point theorem, [24], this will imply that the nonlinear mapping $\left(\phi^{\rho}, \phi^{c}, \phi^{u}, \phi^{v}\right) \in S \rightarrow A\left(\phi^{\rho}, \phi^{c}, \phi^{u}, \phi^{v}\right)$ has a fixed point denoted by $\left(\rho^{\mathrm{DG}}, c^{\mathrm{DG}}, u^{\mathrm{DG}}, v^{\mathrm{DG}}\right)$.

Theorem 5.3 Let the solution of (1.3)-(1.6) satisfy the assumption (5.1). Then for any $\left(\phi^{\rho}, \phi^{c}, \phi^{u}, \phi^{v}\right) \in S, \quad A\left(\phi^{\rho}, \phi^{c}, \phi^{u}, \phi^{v}\right) \in S$.

Proof: Let $\left(\phi^{\rho}, \phi^{c}, \phi^{u}, \phi^{v}\right) \in S$ and $\left(\phi_{L}^{\rho}, \phi_{L}^{c}, \phi_{L}^{u}, \phi_{L}^{v}\right)=A\left(\phi^{\rho}, \phi^{c}, \phi^{u}, \phi^{v}\right)$. We introduce the following notation:

$$
\begin{aligned}
& \tau^{\rho}:=\phi_{L}^{\rho}-\widetilde{\rho}, \quad \xi^{\rho}:=\rho-\widetilde{\rho}, \quad \tau^{c}:=\phi_{L}^{c}-\widetilde{c}, \quad \xi^{c}:=c-\widetilde{c}, \\
& \tau^{u}:=\phi_{L}^{u}-\widetilde{u}, \quad \xi^{u}=u-\widetilde{u}, \quad \tau^{v}:=\phi_{L}^{v}-\widetilde{v}, \quad \xi^{v}:=v-\widetilde{v} .
\end{aligned}
$$

It follows from the consistency Lemma 4.1 that the exact solution of (1.3)-(1.6) satisfies not only equation (3.3) but also the similar equation

$$
\begin{aligned}
& \int_{\Omega} \rho_{t} w^{\rho}+\sum_{E \in \mathcal{E}_{h}} \int_{E} \nabla \rho \nabla w^{\rho}-\sum_{e \in \Gamma_{h}} \int_{e}\left\{\nabla \rho \cdot \mathbf{n}_{e}\right\}\left[w^{\rho}\right]+\varepsilon \sum_{e \in \Gamma_{h}} \int_{e}\left\{\nabla w^{\rho} \cdot \mathbf{n}_{e}\right\}[\rho]+\sigma_{\rho} \sum_{e \in \Gamma_{h}} \frac{r_{\rho}^{2}}{|e|} \int_{e}[\rho]\left[w^{\rho}\right] \\
& -\sum_{E \in \mathcal{E}_{h}} \int_{E} \chi \rho u\left(w^{\rho}\right)_{x}+\sum_{e \in \Gamma_{h}^{\mathrm{ver}}} \int_{e}(\chi \rho u)^{* *} n_{x}\left[w^{\rho}\right]-\sum_{E \in \mathcal{E}_{h}} \int_{E} \chi \rho v\left(w^{\rho}\right)_{y}+\sum_{e \in \Gamma_{h}^{\mathrm{hor}}} \int_{e}(\chi \rho v)^{* *} n_{y}\left[w^{\rho}\right]=0,(5.14)
\end{aligned}
$$

where

$$
\begin{aligned}
(\chi \rho u)^{* *} & :=\frac{a_{L}^{\text {out }}(\chi \rho u)_{e}^{E^{1}}-a_{L}^{\text {in }}(\chi \rho u)_{e}^{E^{2}}}{a_{L}^{\text {out }}-a_{L}^{\text {in }}}-\frac{a_{L}^{\text {out }} a_{L}^{\text {in }}}{a_{L}^{\text {out }}-a_{L}^{\text {in }}}[\rho], \\
(\chi \rho v)^{* *} & :=\frac{b_{L}^{\text {out }}(\chi \rho v)_{e}^{E^{1}}-b_{L}^{\text {in }}(\chi \rho v)_{e}^{E^{2}}}{b_{L}^{\text {out }}-b_{L}^{\text {in }}}-\frac{b_{L}^{\text {out }} b_{L}^{\text {in }}}{b_{L}^{\text {out }}-b_{L}^{\text {in }}}[\rho],
\end{aligned}
$$


and the local speeds $a_{L}^{\text {out }}, a_{L}^{\text {in }}, b_{L}^{\text {out }}$, and $b_{L}^{\text {in }}$ are given by (5.9). Using (5.13), equation (5.14) can be rewritten as:

$$
\begin{aligned}
\int_{\Omega} \widetilde{\rho}_{t} w^{\rho}+ & \sum_{E \in \mathcal{E}_{h}} \int_{E} \nabla \widetilde{\rho} \nabla w^{\rho}-\sum_{e \in \Gamma_{h}} \int_{e}\left\{\nabla \widetilde{\rho} \cdot \mathbf{n}_{e}\right\}\left[w^{\rho}\right]+\varepsilon \sum_{e \in \Gamma_{h}} \int_{e}\left\{\nabla w^{\rho} \cdot \mathbf{n}_{e}\right\}[\widetilde{\rho}]+\sigma_{\rho} \sum_{e \in \Gamma_{h}} \frac{r_{\rho}^{2}}{|e|} \int_{e}[\widetilde{\rho}]\left[w^{\rho}\right] \\
- & \sum_{E \in \mathcal{E}_{h}} \int_{E} \chi \widetilde{\rho} \phi^{u}\left(w^{\rho}\right)_{x}+\sum_{e \in \Gamma_{h}^{\text {ver }}} \int_{e}(\chi \rho u)^{* *} n_{x}\left[w^{\rho}\right]-\sum_{E \in \mathcal{E}_{h}} \int_{E} \chi \widetilde{\rho} \phi^{v}\left(w^{\rho}\right)_{y}+\sum_{e \in \Gamma_{h}^{\text {hor }}} \int_{e}(\chi \rho v)^{* *} n_{y}\left[w^{\rho}\right] \\
= & -\int_{\Omega} \xi_{t}^{\rho} w^{\rho}-\sum_{E \in \mathcal{E}_{h}} \int_{E} \nabla \xi^{\rho} \nabla w^{\rho}+\sum_{e \in \Gamma_{h}} \int_{e}\left\{\nabla \xi^{\rho} \cdot \mathbf{n}_{e}\right\}\left[w^{\rho}\right]-\varepsilon \sum_{e \in \Gamma_{h}} \int_{e}\left\{\nabla w^{\rho} \cdot \mathbf{n}_{e}\right\}\left[\xi^{\rho}\right] \\
& -\sigma_{\rho} \sum_{e \in \Gamma_{h}} \frac{r_{\rho}^{2}}{|e|} \int_{e}\left[\xi^{\rho}\right]\left[w^{\rho}\right]+\sum_{E \in \mathcal{E}_{h}} \int_{E} \chi \xi^{\rho} u\left(w^{\rho}\right)_{x}-\sum_{E \in \mathcal{E}_{h}} \int_{E} \chi \widetilde{\rho}\left(\phi^{u}-u\right)\left(w^{\rho}\right)_{x} \\
& +\sum_{E \in \mathcal{E}_{h}} \int_{E} \chi \xi^{\rho} v\left(w^{\rho}\right)_{y}-\sum_{E \in \mathcal{E}_{h}} \int_{E} \chi \widetilde{\rho}\left(\phi^{v}-v\right)\left(w^{\rho}\right)_{y} .
\end{aligned}
$$

Subtracting equation (5.15) from (5.4) and choosing $w^{\rho}=\tau^{\rho}$, we obtain

$$
\begin{aligned}
\frac{1}{2} \frac{d}{d t}\left(\left\|\tau^{\rho}\right\|_{0, \Omega}\right)+ & \left.\left\|\nabla \tau^{\rho}\right\|\right|_{0, \Omega} ^{2}+\sigma_{\rho} \sum_{e \in \Gamma_{h}} \frac{r_{\rho}^{2}}{|e|}\left\|\left[\tau^{\rho}\right]\right\|_{0, e}^{2} \\
=(1-\varepsilon) & \sum_{e \in \Gamma_{h}} \int_{e}\left\{\nabla \tau^{\rho} \cdot \mathbf{n}_{e}\right\}\left[\tau^{\rho}\right]+\sum_{E \in \mathcal{E}_{h}} \int_{E} \chi \tau^{\rho} \phi^{u}\left(\tau^{\rho}\right)_{x}-\sum_{e \in \Gamma_{h}^{\text {ver }}} \int_{e}\left(\left(\chi \phi_{L}^{\rho} \phi^{u}\right)^{*}-(\chi \rho u)^{* *}\right) n_{x}\left[\tau^{\rho}\right] \\
& +\sum_{E \in \mathcal{E}_{h}} \int_{E} \chi \tau^{\rho} \phi^{v}\left(\tau^{\rho}\right)_{y}-\sum_{e \in \Gamma_{h}^{\text {hor }}} \int_{e}\left(\left(\chi \phi_{L}^{\rho} \phi^{v}\right)^{*}-(\chi \rho v)^{* *}\right) n_{y}\left[\tau^{\rho}\right] \\
& +\int_{\Omega} \xi_{t}^{\rho} \tau^{\rho}+\sum_{E \in \mathcal{E}_{h}} \int_{E} \nabla \xi^{\rho} \nabla \tau^{\rho}-\sum_{e \in \Gamma_{h}} \int_{e}\left\{\nabla \xi^{\rho} \cdot \mathbf{n}_{e}\right\}\left[\tau^{\rho}\right]+\varepsilon \sum_{e \in \Gamma_{h}} \int_{e}\left\{\nabla \tau^{\rho} \cdot \mathbf{n}_{e}\right\}\left[\xi^{\rho}\right] \\
& +\sigma_{\rho} \sum_{e \in \Gamma_{h}} \frac{r_{\rho}^{2}}{|e|} \int_{e}\left[\xi^{\rho}\right]\left[\tau^{\rho}\right]-\sum_{E \in \mathcal{E}_{h}} \int_{E} \chi \xi^{\rho} u\left(\tau^{\rho}\right)_{x}-\sum_{E \in \mathcal{E}_{h}} \int_{E} \chi \xi^{\rho} v\left(\tau^{\rho}\right)_{y} \\
& +\sum_{E \in \mathcal{E}_{h}} \int_{E} \chi \widetilde{\rho}\left(\phi^{u}-u\right)\left(\tau^{\rho}\right)_{x}+\sum_{E \in \mathcal{E}_{h}} \int_{E} \chi \widetilde{\rho}\left(\phi^{v}-v\right)\left(\tau^{\rho}\right)_{y}=: T_{1}^{\rho}+T_{2}^{\rho}+\ldots+T_{14}^{\rho} .
\end{aligned}
$$

Next, we bound each term on the RHS of (5.16) using standard DG techniques. The quantities $\varepsilon_{i}$ in the estimates below are positive real numbers, which will be defined later.

We begin with the first term on the RHS of (5.16). The Cauchy-Schwarz inequality yields:

$$
\left|T_{1}^{\rho}\right| \leq(1-\varepsilon) \sum_{e \in \Gamma_{h}}\left\|\left\{\nabla \tau^{\rho}\right\}\right\|_{0, e}\left\|\left[\tau^{\rho}\right]\right\|_{0, e} .
$$

As before, we denote by $E^{1}$ and $E^{2}$ the two elements sharing the edge $e$. Then, using the inequality (2.5), we obtain

$$
\sum_{e \in \Gamma_{h}}\left\|\left\{\nabla \tau^{\rho}\right\}\right\|_{0, e}\left\|\left[\tau^{\rho}\right]\right\|_{0, e} \leq \sum_{e \in \Gamma_{h}} \frac{1}{2}\left(\left\|\left(\nabla \tau^{\rho}\right)_{e}^{E^{1}}\right\|_{0, e}+\left\|\left(\nabla \tau^{\rho}\right)_{e}^{E^{2}}\right\|_{0, e}\right)\left\|\left[\tau^{\rho}\right]\right\|_{0, e}
$$




$$
\leq \frac{C_{t} r_{\rho}}{2 \sqrt{h}} \sum_{e \in \Gamma_{h}}\left(\left\|\nabla \tau^{\rho}\right\|_{0, E^{1}}+\left\|\nabla \tau^{\rho}\right\|_{0, E^{2}}\right)\left\|\left[\tau^{\rho}\right]\right\|_{0, e}
$$

and hence, using the fact that $|e| \leq \sqrt{h}$, we end up with the following bound on $T_{1}^{\rho}$ :

$$
\left|T_{1}^{\rho}\right| \leq \varepsilon_{1}^{\rho} \sum_{E \in \mathcal{E}_{h}}\left\|\nabla \tau^{\rho}\right\|_{0, E}^{2}+C_{1}^{\rho} \sum_{e \in \Gamma_{h}} \frac{r_{\rho}^{2}}{|e|}\left\|\left[\tau^{\rho}\right]\right\|_{0, e}^{2}=\varepsilon_{1}^{\rho} \mid\left\|\nabla \tau^{\rho}\right\|\left\|_{0, \Omega}^{2}+C_{1}^{\rho} \sum_{e \in \Gamma_{h}} \frac{r_{\rho}^{2}}{|e|}\right\|\left[\tau^{\rho}\right] \|_{0, e}^{2} .
$$

Consider now the second term on the RHS of (5.16). From Lemma 5.1 we know that $\phi^{u}$ is a bounded function, hence $T_{2}^{\rho}$ can be bounded as follows:

$$
\left|T_{2}^{\rho}\right| \leq \varepsilon_{2}^{\rho} \sum_{E \in \mathcal{E}_{h}}\left\|\left(\tau^{\rho}\right)_{x}\right\|_{0, E}^{2}+C_{2}^{\rho}\left\|\tau^{\rho}\right\|_{0, \Omega}^{2} \leq \varepsilon_{2}^{\rho}\|\|\left(\tau^{\rho}\right)_{x}\|\|_{0, \Omega}^{2}+C_{2}^{\rho}\left\|\tau^{\rho}\right\|_{0, \Omega}^{2}
$$

Next, we bound the third term on the RHS of (5.16) as

$$
\begin{aligned}
\left|T_{3}^{\rho}\right| \leq \sum_{e \in \Gamma_{h}^{\text {ver }}} & \left(\left|\int_{e} \frac{a_{L}^{\text {out }}}{a_{L}^{\text {out }}-a_{L}^{\text {in }}}\left(\left(\chi \phi_{L}^{\rho} \phi^{u}\right)_{e}^{E^{1}}-(\chi \rho u)_{e}^{E^{1}}\right) n_{x}\left[\tau^{\rho}\right]\right|\right. \\
+ & \left|\int_{e} \frac{-a_{L}^{\text {in }}}{a_{L}^{\text {out }}-a_{L}^{\text {in }}}\left(\left(\chi \phi_{L}^{\rho} \phi^{u}\right)_{e}^{E^{2}}-(\chi \rho u)_{e}^{E^{2}}\right) n_{x}\left[\tau^{\rho}\right]\right| \\
+ & \left.\left|\int_{e} \frac{-a_{L}^{\text {in }} a_{L}^{\text {out }}}{a_{L}^{\text {out }}-a_{L}^{\text {in }}}\left[\phi_{L}^{\rho}-\rho\right] n_{x}\left[\tau^{\rho}\right]\right|\right)=: \mathrm{I}+\text { II }+ \text { III. }
\end{aligned}
$$

Using (5.10) and (5.13), the first term on the RHS of (5.19) can be estimated by

$$
\begin{aligned}
& \mathrm{I} \leq \chi \sum_{e \in \Gamma_{h}^{\text {ver }}}\left|\int_{e}\left(\left(\phi_{L}^{\rho} \phi^{u}\right)_{e}^{E^{1}}-(\rho u)_{e}^{E^{1}}\right) n_{x}\left[\tau^{\rho}\right]\right| \\
& \leq \chi \sum_{e \in \Gamma_{h}^{\text {ver }}}\left(\left|\int_{e}\left(\tau^{\rho} \phi^{u}\right)_{e}^{E^{1}} n_{x}\left[\tau^{\rho}\right]\right|+\left|\int_{e}\left(\xi^{\rho} \phi^{u}\right)_{e}^{E^{1}} n_{x}\left[\tau^{\rho}\right]\right|\right. \\
& \left.+\left|\int_{e}\left(\left(\phi^{u}-\widetilde{u}\right) \rho\right)_{e}^{E^{1}} n_{x}\left[\tau^{\rho}\right]\right|+\left|\int_{e}\left(\xi^{u} \rho\right)_{e}^{E^{1}} n_{x}\left[\tau^{\rho}\right]\right|\right)=: \widetilde{\mathrm{I}} .
\end{aligned}
$$

We now use the Cauchy-Schwarz inequality, the trace inequality (2.3), the inequality (2.5), the assumption (3.2), the approximation inequality (2.2), and the bound on $\phi^{u}$ from Lemma 5.1 to obtain the bound on $\widetilde{\mathrm{I}}$ :

$$
\widetilde{\mathrm{I}} \leq \frac{1}{2}\left\|\tau^{\rho}\right\|_{0, \Omega}^{2}+K \sum_{e \in \Gamma_{h}} \frac{r_{\rho}^{2}}{|e|}\left\|\left[\tau^{\rho}\right]\right\|_{0, e}^{2}+C^{*}\left(\frac{h^{2 \min \left(r_{\rho}+1, s_{\rho}\right)}}{r_{\rho}^{2 s_{\rho}}}+\frac{h^{2 \min \left(r_{u}+1, s_{u}\right)}}{r_{u}^{2 s_{u}}}\right)+C^{* *}\left\|\phi^{u}-\widetilde{u}\right\|_{0, \Omega}^{2} .
$$

A similar bound can be derived for the second term II on the RHS of (5.19). To estimate the last term on the RHS of (5.19), we first use (5.13) and the definition of the one-sided local speeds (5.9) to obtain

$$
\mathrm{III} \leq C \sum_{e \in \Gamma_{h}^{\text {ver }}}\left(\left\|\left[\tau^{\rho}\right]\right\|_{0, e}^{2}+\left|\int_{e}\left[\xi^{\rho}\right]\left[\tau^{\rho}\right]\right|\right):=\widetilde{\mathrm{III}}
$$


Then, using the Cauchy-Schwarz inequality, the trace inequality (2.3), and the approximation inequality (2.2), we bound $\widetilde{I I I}$ as follows:

$$
\widetilde{\mathrm{III}} \leq\left(\frac{K_{1} h}{r_{\rho}^{2}}+K_{2}\right) \sum_{e \in \Gamma_{h}} \frac{r_{\rho}^{2}}{|e|}\left\|\left[\tau^{\rho}\right]\right\|_{0, e}^{2}+C \frac{h^{2 \min \left(r_{\rho}+1, s_{\rho}\right)}}{r_{\rho}^{2 s_{\rho}}} .
$$

Combining the above bounds on I, II, and III, we arrive at

$$
\left|T_{3}^{\rho}\right| \leq\left\|\tau^{\rho}\right\|_{0, \Omega}^{2}+C_{3}^{\rho} \sum_{e \in \Gamma_{h}} \frac{r_{\rho}^{2}}{|e|}\left\|\left[\tau^{\rho}\right]\right\|_{0, e}^{2}+C^{*}\left(\frac{h^{2 \min \left(r_{\rho}+1, s_{\rho}\right)}}{r_{\rho}^{2 s_{\rho}}}+\frac{h^{2 \min \left(r_{u}+1, s_{u}\right)}}{r_{u}^{2 s_{u}}}\right)+C^{* *}\left\|\phi^{u}-\widetilde{u}\right\|_{0, \Omega}^{2} .
$$

The terms $T_{4}^{\rho}$ and $T_{5}^{\rho}$ are bounded in the same way as the terms $T_{2}^{\rho}$ and $T_{3}^{\rho}$, respectively, and the bounds are:

$$
\left|T_{4}^{\rho}\right| \leq \varepsilon_{2}^{\rho}\left|\left\|\left(\tau^{\rho}\right)_{y} \mid\right\|_{0, \Omega}^{2}+C_{4}^{\rho}\left\|\tau^{\rho}\right\|_{0, \Omega}^{2}\right.
$$

and

$$
\left|T_{5}^{\rho}\right| \leq\left\|\tau^{\rho}\right\|_{0, \Omega}^{2}+C_{5}^{\rho} \sum_{e \in \Gamma_{h}} \frac{r_{\rho}^{2}}{|e|}\left\|\left[\tau^{\rho}\right]\right\|_{0, e}^{2}+C^{*}\left(\frac{h^{2 \min \left(r_{\rho}+1, s_{\rho}\right)}}{r_{\rho}^{2 s_{\rho}}}+\frac{h^{2 \min \left(r_{v}+1, s_{v}\right)}}{r_{v}^{2 s_{v}}}\right)+C^{* *}\left\|\phi^{v}-\widetilde{v}\right\|_{0, \Omega}^{2} .
$$

The term $T_{6}^{\rho}$ is bounded using the Cauchy-Schwarz inequality and the approximation inequality (2.2):

$$
\left|T_{6}^{\rho}\right| \leq\left\|\tau^{\rho}\right\|_{0, \Omega}^{2}+C^{*} \frac{h^{2 \min \left(r_{\rho}+1, s_{\rho}\right)}}{r_{\rho}^{2 s_{\rho}}} .
$$

Using the Cauchy-Schwarz inequality, Young's inequality, and the approximation inequality (2.2) for $\rho$, we obtain the following bound for the term $T_{7}^{\rho}$ :

$$
\left|T_{7}^{\rho}\right| \leq\left.\varepsilon_{7}^{\rho}||\left|\nabla \tau^{\rho}\right|\right|_{0, \Omega} ^{2}+C^{*} \frac{h^{2 \min \left(r_{\rho}+1, s_{\rho}\right)-2}}{r_{\rho}^{2 s_{\rho}-2}} .
$$

The term $T_{8}^{\rho}$ is bounded using the Cauchy-Schwarz inequality, the trace inequality (2.4), and the approximation inequality $(2.2)$ :

$$
\left|T_{8}^{\rho}\right| \leq C_{8}^{\rho} \sum_{e \in \Gamma_{h}} \frac{r_{\rho}^{2}}{|e|}\left\|\left[\tau^{\rho}\right]\right\|_{0, e}^{2}+C^{*} \frac{h^{2 \min \left(r_{\rho}+1, s_{\rho}\right)-2}}{r_{\rho}^{2 s_{\rho}-2}} .
$$

To bound the term $T_{9}^{\rho}$ we use the trace inequality (2.5), inequality (2.3), the Cauchy-Schwarz inequality and Young's inequality:

$$
\left|T_{9}^{\rho}\right| \leq\left.\varepsilon_{9}^{\rho}||\left|\nabla \tau^{\rho}\right|\right|_{0, \Omega} ^{2}+C^{*} \frac{h^{2 \min \left(r_{\rho}+1, s_{\rho}\right)-2}}{r_{\rho}^{2 s_{\rho}-4}} .
$$

Similarly, we bound the term $T_{10}^{\rho}$ by:

$$
\left|T_{10}^{\rho}\right| \leq C_{10}^{\rho} \sum_{e \in \Gamma_{h}} \frac{r_{\rho}^{2}}{|e|}\left\|\left[\tau^{\rho}\right]\right\|_{0, e}^{2}+C^{*} \frac{h^{2 \min \left(r_{\rho}+1, s_{\rho}\right)-2}}{r_{\rho}^{2 s_{\rho}-4}} .
$$


For the terms $T_{11}^{\rho}$ and $T_{12}^{\rho}$, we use our assumption on the smoothness of the exact solution together with the Cauchy-Schwarz inequality and the approximation inequality (2.2) to obtain the following bounds:

$$
\left|T_{11}^{\rho}\right| \leq \varepsilon_{11}^{\rho}||\left|\left(\tau^{\rho}\right)_{x}\right|||_{0, \Omega}^{2}+C^{*} \frac{h^{2 \min \left(r_{\rho}+1, s_{\rho}\right)}}{r_{\rho}^{2 s_{\rho}}}, \quad\left|T_{12}^{\rho}\right| \leq \varepsilon_{11}^{\rho}\left|\left\|\left|\left(\tau^{\rho}\right)_{y}\right|\right\|_{0, \Omega}^{2}+C^{*} \frac{h^{2 \min \left(r_{\rho}+1, s_{\rho}\right)}}{r_{\rho}^{2 s_{\rho}}}\right.
$$

Consider now the term $T_{13}^{\rho}$. We first use (5.13) to obtain

$$
\left|T_{13}^{\rho}\right| \leq C \sum_{E \in \mathcal{E}_{h}}\left(\left|\int_{E}\left(\phi^{u}-\widetilde{u}\right)\left(\tau^{\rho}\right)_{x}\right|+\left|\int_{E} \xi^{u}\left(\tau^{\rho}\right)_{x}\right|\right) .
$$

Then we apply the Cauchy-Schwarz inequality and the approximation inequality (2.2), which result in

$$
\left|T_{13}^{\rho}\right| \leq \varepsilon_{13}^{\rho}\left|\left\|\left(\tau^{\rho}\right)_{x} \mid\right\|_{0, \Omega}^{2}+C^{*} \frac{h^{2 \min \left(r_{u}+1, s_{u}\right)}}{r_{u}^{2 s_{u}}}+C^{* *}\left\|\phi^{u}-\widetilde{u}\right\|_{0, \Omega}^{2} .\right.
$$

The bound on the term $T_{14}^{\rho}$ is obtained in the same way as the bound on $T_{13}^{\rho}$ :

$$
\left|T_{14}^{\rho}\right| \leq \varepsilon_{13}^{\rho}\left|\left\|\left(\tau^{\rho}\right)_{y}||_{0, \Omega}^{2}+C^{*} \frac{h^{2 \min \left(r_{v}+1, s_{v}\right)}}{r_{v}^{2 s_{v}}}+C^{* *}\right\| \phi^{v}-\widetilde{v} \|_{0, \Omega}^{2}\right.
$$

Finally, we plug the estimates (5.17)-(5.18) and (5.20)-(5.30) into (5.16) and use the assumption that $h<1$ to obtain

$$
\begin{aligned}
\frac{1}{2} \frac{d}{d t}\left\|\tau^{\rho}\right\|_{0, \Omega}^{2}+ & \left(1-\varepsilon_{1}^{\rho}-\varepsilon_{2}^{\rho}-\varepsilon_{7}^{\rho}-\varepsilon_{9}^{\rho}-\varepsilon_{11}^{\rho}-\varepsilon_{13}^{\rho}\right)\|\| \nabla \tau^{\rho}\|\|_{0, \Omega}^{2} \\
+ & \left(\sigma_{\rho}-C_{1}^{\rho}-C_{3}^{\rho}-C_{5}^{\rho}-C_{8}^{\rho}-C_{10}^{\rho}\right) \sum_{e \in \Gamma_{h}} \frac{r_{\rho}^{2}}{|e|}\left\|\left[\tau^{\rho}\right]\right\|_{0, \Omega}^{2} \\
\leq & \left(3+C_{2}^{\rho}+C_{4}^{\rho}\right)\left\|\tau^{\rho}\right\|_{0, \Omega}^{2}+C_{\rho}^{*}\left(\frac{h^{2 \min \left(r_{\rho}+1, s_{\rho}\right)-2}}{r_{\rho}^{2 s_{\rho}-4}}+\frac{h^{2 \min \left(r_{u}+1, s_{u}\right)}}{r_{u}^{2 s_{u}}}+\frac{h^{2 \min \left(r_{v}+1, s_{v}\right)}}{r_{v}^{2 s_{v}}}\right) \\
& +C^{* *}\left(\left\|\phi^{u}-\widetilde{u}\right\|_{0, \Omega}^{2}+\left\|\phi^{v}-\widetilde{v}\right\|_{0, \Omega}^{2}\right) .
\end{aligned}
$$

We now choose $\varepsilon_{i}^{\rho}$ and the penalty parameter $\sigma_{\rho}$ so that the coefficients of the $\left\|\mid \nabla \tau^{\rho}\right\| \|_{0, \Omega}^{2}$ and $\sum_{e \in \Gamma_{h}} \frac{r_{\rho}^{2}}{|e|}\left\|\left[\tau^{\rho}\right]\right\|_{0, \Omega}^{2}$ on the LHS of (5.31) are equal to $1 / 2$. We then multiply equation (5.31) by 2 and integrate it in time from 0 to $t$. Taking into account that $\left(\phi^{u}, \phi^{v}\right) \in S$ and using the fact that $\tau^{0}=0$, we obtain:

$$
\begin{aligned}
& \left\|\tau^{\rho}\right\|_{0, \Omega}^{2}+\int_{0}^{t}\left(\left\|\mid \nabla \tau^{\rho}\right\|\left\|_{0, \Omega}^{2}+\sum_{e \in \Gamma_{h}} \frac{r_{\rho}^{2}}{|e|}\right\|\left[\tau^{\rho} \|_{0, e}^{2}\right) \leq \widetilde{C}^{\rho} \int_{0}^{t}\left\|\tau^{\rho}\right\|_{0, \Omega}^{2}\right. \\
& \quad+C^{u v}\left(\frac{h^{2 \min \left(r_{\rho}+1, s_{\rho}\right)-2}}{r_{\rho}^{2 s_{\rho}-4}}+\frac{h^{2 \min \left(r_{c}+1, s_{c}\right)-2}}{r_{c}^{2 s_{c}-4}}+\frac{h^{2 \min \left(r_{u}+1, s_{u}\right)-2}}{r_{u}^{2 s_{u}-4}}+\frac{h^{2 \min \left(r_{v}+1, s_{v}\right)-2}}{r_{v}^{2 s_{v}-4}}\right) .
\end{aligned}
$$


Next, we apply Gronwall's Lemma 2.6 and take the supremum with respect to $t$ of the both sides of (5.32):

$$
\begin{aligned}
& \sup _{[0, T]}\left\|\tau^{\rho}\right\|_{0, \Omega}^{2}+\int_{0}^{T}\left(\left\|\nabla \tau^{\rho}\right\|\left\|_{0, \Omega}^{2}+\sum_{e \in \Gamma_{h}} \frac{r_{\rho}^{2}}{|e|}\right\|\left[\tau^{\rho}\right] \|_{0, e}^{2}\right) \\
& \leq C^{\mathrm{I}}\left(\frac{h^{2 \min \left(r_{\rho}+1, s_{\rho}\right)-2}}{r_{\rho}^{2 s_{\rho}-4}}+\frac{h^{2 \min \left(r_{c}+1, s_{c}\right)-2}}{r_{c}^{2 s_{c}-4}}+\frac{h^{2 \min \left(r_{u}+1, s_{u}\right)-2}}{r_{u}^{2 s_{u}-4}}+\frac{h^{2 \min \left(r_{v}+1, s_{v}\right)-2}}{r_{v}^{2 s_{v}-4}}\right)
\end{aligned}
$$

where $C^{\mathrm{I}}$ is a constant that depends on $\|\rho\|_{\left(L^{\infty}([0, T]) ; H^{2}(\Omega)\right)},\left\|\rho_{t}\right\|_{\left(L^{\infty}([0, T]) ; L^{2}(\Omega)\right)},\|u\|_{\left(L^{\infty}([0, T]) ; L^{2}(\Omega)\right)}$, $\|v\|_{\left(L^{\infty}([0, T]) ; L^{2}(\Omega)\right)}$, and $T$ only.

According to the definition on page 9, the estimate (5.33) implies that $\phi_{L}^{\rho} \in S$.

Using similar techniques, it can be shown that $\left(\phi_{L}^{c}, \phi_{L}^{u}, \phi_{L}^{v}\right) \in S$ as well (see Appendix A for the detailed proof). Therefore, we have shown that $A(S) \subset S$, and the proof of Theorem 5.3 is now complete.

Let us recall that our goal is to show that the operator $A$ has a fixed point. Equipped with Theorem 5.3, it remained to prove that $A$ is compact. To this end, we need to show that $A$ is continuous and equicontinuous.

Lemma 5.4 The operator $A$ is continuous and equicontinuous.

Proof: We consider the sequence $\left\{\left(\phi_{n}^{\rho}, \phi_{n}^{c}, \phi_{n}^{u}, \phi_{n}^{v}\right)\right\}$ and assume that

$$
\sup _{t \in[0, T]}\left(\left\|\left(\phi_{n}^{\rho}, \phi_{n}^{c}, \phi_{n}^{u}, \phi_{n}^{v}\right)-\left(\phi^{\rho}, \phi^{c}, \phi^{u}, \phi^{v}\right)\right\|_{S}\right) \rightarrow 0 \quad \text { as } \quad n \rightarrow \infty .
$$

Let

$$
\left(\phi_{L, n}^{\rho}, \phi_{L, n}^{c}, \phi_{L, n}^{u}, \phi_{L, n}^{v}\right)=A\left(\phi_{n}^{\rho}, \phi_{n}^{c}, \phi_{n}^{u}, \phi_{n}^{v}\right)
$$

and

$$
\left(\phi_{L}^{\rho}, \phi_{L}^{c}, \phi_{L}^{u}, \phi_{L}^{v}\right)=A\left(\phi^{\rho}, \phi^{c}, \phi^{u}, \phi^{v}\right)
$$

be two solutions of (5.4)-(5.7). We denote by $\left(\widehat{\phi}_{L}^{\rho}, \widehat{\phi}_{L}^{c}, \widehat{\phi}_{L}^{u}, \widehat{\phi}_{L}^{v}\right)$ the difference between these two solutions (note that $\left.\left(\widehat{\phi}_{L}^{\rho, 0}, \widehat{\phi}_{L}^{c, 0}, \widehat{\phi}_{L}^{u, 0}, \widehat{\phi}_{L}^{v, 0}\right)=(0,0,0,0)\right)$, subtract (5.35) from (5.34), and choose the test function in the resulting equation for $\rho$ to be $w^{\rho}=\widehat{\phi}_{L}^{\rho}$. This yields:

$$
\begin{aligned}
\frac{1}{2} \frac{d}{d t}\left\|\widehat{\phi}_{L}^{\rho}\right\|_{0, \Omega}^{2}+\left\|\left|\nabla \widehat{\phi}_{L}^{\rho}\left\|\left.\right|_{0, \Omega} ^{2}+\sigma_{\rho} \sum_{e \in \Gamma_{h}} \frac{r_{\rho}^{2}}{|e|}\right\|\left[\widehat{\phi}_{L}^{\rho}\right] \|_{0, e}^{2}\right.\right. \\
=(1-\varepsilon) \sum_{e \in \Gamma_{h}} \int_{e}\left\{\nabla \widehat{\phi}_{L}^{\rho} \cdot \mathbf{n}_{e}\right\}\left[\widehat{\phi}_{L}^{\rho}\right]+\sum_{E \in \mathcal{E}_{h}} \int_{E} \chi \widehat{\phi}_{L}^{\rho} \phi^{u}\left(\widehat{\phi}_{L}^{\rho}\right)_{x}+\sum_{E \in \mathcal{E}_{h}} \int_{E} \chi \phi_{L, n}^{\rho}\left(\phi_{n}^{u}-\phi^{u}\right)\left(\widehat{\phi}_{L}^{\rho}\right)_{x} \\
\quad-\sum_{e \in \Gamma_{h}^{\text {ver }}} \int_{e}\left(\chi \widehat{\phi}_{L}^{\rho} \phi^{u}\right)^{*} n_{x}\left[\widehat{\phi}_{L}^{\rho}\right]+\sum_{e \in \Gamma_{h}^{\text {ver }}} \int_{e}\left(\left(\chi \phi_{L, n}^{\rho} \phi^{u}\right)^{*}-\left(\chi \phi_{L, n}^{\rho} \phi_{n}^{u}\right)^{*}\right) n_{x}\left[\widehat{\phi}_{L}^{\rho}\right] \\
\quad+\sum_{E \in \mathcal{E}_{h}} \int_{E} \chi \widehat{\phi}_{L}^{\rho} \phi^{v}\left(\widehat{\phi}_{L}^{\rho}\right)_{y}+\sum_{E \in \mathcal{E}_{h}} \int_{E} \chi \phi_{L, n}^{\rho}\left(\phi_{n}^{v}-\phi^{v}\right)\left(\widehat{\phi}_{L}^{\rho}\right)_{y}-\sum_{e \in \Gamma_{h}^{\text {hor }}} \int_{e}\left(\chi \widehat{\phi}_{L}^{\rho} \phi^{v}\right)^{*} n_{y}\left[\widehat{\phi}_{L}^{\rho}\right] \\
\left.\quad+\sum_{e \in \Gamma_{h}^{\text {hor }}} \int_{e}\left(\left(\chi \phi_{L, n}^{\rho} \phi^{v}\right)^{*}-\left(\chi \phi_{L, n}^{\rho} \phi_{n}^{v}\right)^{*}\right)\right) n_{y}\left[\widehat{\phi}_{L}^{\rho}\right]=: R_{1}+R_{2}+\ldots+R_{9} .
\end{aligned}
$$


We now bound each term on the RHS of (5.36).

The term $R_{1}$ can be bounded using the Cauchy-Schwarz inequality, Young's inequality, and the inequality (2.5):

$$
\left|R_{1}\right| \leq \frac{1}{6}\left\|\left|\nabla \widehat{\phi}_{L}^{\rho}\right|\right\|_{0, \Omega}^{2}+C_{1} \sum_{e \in \Gamma_{h}} \frac{r_{\rho}^{2}}{|e|}\left\|\left[\widehat{\phi}_{L}^{\rho}\right]\right\|_{0, e}^{2} .
$$

Next, applying the Cauchy-Schwarz and Young's inequalities and using the boundedness of $\left\|\phi^{u}\right\|_{\infty, \Omega}$, established in Lemma 5.1, we obtain the following bound on $R_{2}$ :

$$
\left|R_{2}\right| \leq \frac{1}{6}\left\|\mid\left(\widehat{\phi}_{L}^{\rho}\right)_{x}\right\|\left\|_{0, \Omega}^{2}+C_{2}\right\| \widehat{\phi}_{L}^{\rho} \|_{0, \Omega}^{2}
$$

Using the Cauchy-Schwarz and Young's inequalities and the fact that $\phi_{L, n}^{\rho} \in S$, we bound the term $R_{3}$ by

$$
\left|R_{3}\right| \leq \frac{1}{6}\left\|( \widehat { \phi } _ { L } ^ { \rho } ) _ { x } \left|\left\|\left.\right|_{0, \Omega} ^{2}+C_{3}\right\| \phi_{n}^{u}-\phi^{u} \|_{0, \Omega}^{2}\right.\right.
$$

We then use the Cauchy-Schwarz inequality, the inequality (2.5), and the first numerical flux formula in (5.8) to estimate $R_{4}$ :

$$
\left|R_{4}\right| \leq\left\|\widehat{\phi}_{L}^{\rho}\right\|_{0, \Omega}^{2}+C_{4} \sum_{e \in \Gamma_{h}} \frac{r_{\rho}^{2}}{|e|}\left\|\left[\widehat{\phi}_{L}^{\rho}\right]\right\|_{0, e}^{2} .
$$

We now consider the term $R_{5}$. It follows from formulae (5.8)-(5.9) that the numerical fluxes $\left(\chi \phi_{L, n}^{\rho} \phi^{u}\right)^{*}$ is the composition of the continuous functions with respect to the variables $\left(\phi^{u}\right)_{e}^{E^{1}}$ and $\left(\phi^{u}\right)_{e}^{E^{2}}$. Hence, we can apply the Cauchy-Schwarz inequality and the inequality (2.5) to $R_{5}$ so that it is bounded by

$$
\left|R_{5}\right| \leq\left\|\left(\chi \phi_{L, n}^{\rho} \phi^{u}\right)^{*}-\left(\chi \phi_{L, n}^{\rho} \phi_{n}^{u}\right)^{*}\right\|_{0, \Omega}^{2}+C_{5} \sum_{e \in \Gamma_{h}} \frac{r_{\rho}^{2}}{|e|}\left\|\left[\widehat{\phi}_{L}^{\rho}\right]\right\|_{0, e}^{2} .
$$

The terms $R_{6}, R_{7}, R_{8}$, and $R_{9}$ are similar to the terms $R_{2}, R_{3}, R_{4}$, and $R_{5}$ estimated in (5.38), (5.39), (5.40), and (5.41), respectively. Therefore, we obtain

$$
\begin{aligned}
& \left|R_{6}\right| \leq \frac{1}{6}\left\|\left|\left(\widehat{\phi}_{L}^{\rho}\right)_{y}\left\|\left.\right|_{0, \Omega} ^{2}+C_{6}\right\| \widehat{\phi}_{L}^{\rho} \|_{0, \Omega}^{2},\right.\right. \\
& \left|R_{7}\right| \leq \frac{1}{6}\left\|\left|\left(\widehat{\phi}_{L}^{\rho}\right)_{y}\left\|\left.\right|_{0, \Omega} ^{2}+C_{7}\right\| \phi_{n}^{v}-\phi^{v} \|_{0, \Omega}^{2},\right.\right. \\
& \left|R_{8}\right| \leq\left\|\widehat{\phi}_{L}^{\rho}\right\|_{0, \Omega}^{2}+C_{8} \sum_{e \in \Gamma_{h}} \frac{r_{\rho}^{2}}{|e|}\left\|\left[\widehat{\phi}_{L}^{\rho}\right]\right\|_{0, e}^{2}, \\
& \left|R_{9}\right| \leq\left\|\left(\chi \phi_{L, n}^{\rho} \phi^{v}\right)^{*}-\left(\chi \phi_{L, n}^{\rho} \phi_{n}^{v}\right)^{*}\right\|_{0, \Omega}^{2}+C_{9} \sum_{e \in \Gamma_{h}} \frac{r_{\rho}^{2}}{|e|}\left\|\left[\widehat{\phi}_{L}^{\rho}\right]\right\|_{0, e}^{2} .
\end{aligned}
$$

Substituting the estimates (5.37)-(5.45) into (5.36) yields:

$$
\begin{gathered}
\frac{1}{2} \frac{d}{d t}\left\|\widehat{\phi}_{L}^{\rho}\right\|_{0, \Omega}^{2}+\frac{1}{2}\|\| \nabla \widehat{\phi}_{L}^{\rho}\left\|\left.\right|_{0, \Omega} ^{2}+\left(\sigma_{\rho}-C\right) \sum_{e \in \Gamma_{h}} \frac{r_{\rho}^{2}}{|e|}\right\|\left[\widehat{\phi}_{L}^{\rho}\right]\left\|_{0, e}^{2} \leq C^{*}\right\| \widehat{\phi}_{L}^{\rho} \|_{0, \Omega}^{2}+C^{* *}\left(\left\|\phi_{n}^{u}-\phi^{u}\right\|_{0, \Omega}^{2}\right. \\
\left.+\left\|\phi_{n}^{v}-\phi^{v}\right\|_{0, \Omega}^{2}+\left\|\left(\chi \phi_{L, n}^{\rho} \phi^{u}\right)^{*}-\left(\chi \phi_{L, n}^{\rho} \phi_{n}^{u}\right)^{*}\right\|_{0, \Omega}^{2}+\left\|\left(\chi \phi_{L, n}^{\rho} \phi^{v}\right)^{*}-\left(\chi \phi_{L, n}^{\rho} \phi_{n}^{v}\right)^{*}\right\|_{0, \Omega}^{2}\right)
\end{gathered}
$$


where the penalty parameter $\sigma_{\rho}$ is chosen sufficiently large so that the coefficient $\left(\sigma_{\rho}-C\right)$ is nonnegative.

We now integrate the latter inequality with respect to time from 0 to $t$ and apply Gronwall's Lemma 2.6 to obtain

$$
\begin{aligned}
\left\|\widehat{\phi}_{L}^{\rho}\right\|_{0, \Omega}^{2} & +\int_{0}^{t}\left(\left\|\nabla \widehat{\phi}_{L}^{\rho} \mid\right\|_{0, \Omega}^{2}+\left(\sigma_{\rho}-C\right) \sum_{e \in \Gamma_{h}} \frac{r_{\rho}^{2}}{|e|}\left\|\left[\widehat{\phi}_{L}^{\rho}\right]\right\|_{0, e}^{2}\right) d t \leq M\left(\left\|\phi_{L}^{\rho, 0}\right\|_{0, \Omega}^{2}+\int_{0}^{t}\left(\left\|\phi_{n}^{u}-\phi^{u}\right\|_{0, \Omega}^{2}\right.\right. \\
& \left.\left.+\left\|\phi_{n}^{v}-\phi^{v}\right\|_{0, \Omega}^{2}+\left\|\left(\chi \phi_{L, n}^{\rho} \phi^{u}\right)^{*}-\left(\chi \phi_{L, n}^{\rho} \phi_{n}^{u}\right)^{*}\right\|_{0, \Omega}^{2}+\left\|\left(\chi \phi_{L, n}^{\rho} \phi^{v}\right)^{*}-\left(\chi \phi_{L, n}^{\rho} \phi_{n}^{v}\right)^{*}\right\|_{0, \Omega}^{2}\right)\right) .
\end{aligned}
$$

Finally, taking the supremum over $t$ and since $\widehat{\phi}_{L}^{\rho, 0}=0$, we arrive at

$$
\begin{aligned}
\sup _{t \in[0, T]}\left\|\widehat{\phi}_{L}^{\rho}\right\|_{0, \Omega}^{2} & +\int_{0}^{T}\left(\left\|\nabla \widehat{\phi}_{L}^{\rho}\right\|\left\|_{0, \Omega}^{2}+\sum_{e \in \Gamma_{h}} \frac{r_{\rho}^{2}}{|e|}\right\|\left[\widehat{\phi}_{L}^{\rho}\right] \|_{0, e}^{2}\right) d t \leq M^{*} \int_{0}^{T}\left(\left\|\phi_{n}^{u}-\phi^{u}\right\|_{0, \Omega}^{2}+\left\|\phi_{n}^{v}-\phi^{v}\right\|_{0, \Omega}^{2}\right. \\
& \left.+\left\|\left(\chi \phi_{L, n}^{\rho} \phi^{u}\right)^{*}-\left(\chi \phi_{L, n}^{\rho} \phi_{n}^{u}\right)^{*}\right\|_{0, \Omega}^{2}+\left\|\left(\chi \phi_{L, n}^{\rho} \phi^{v}\right)^{*}-\left(\chi \phi_{L, n}^{\rho} \phi_{n}^{v}\right)^{*}\right\|_{0, \Omega}^{2}\right) .
\end{aligned}
$$

This inequality together with the similar inequalities for $\widehat{\phi}^{c}, \widehat{\phi}^{u}$, and $\widehat{\phi}^{v}$, which can be obtained in an analogous way, imply continuity of the operator A.

Applying similar techniques to the difference $\left(\bar{\phi}_{L}^{\rho}, \bar{\phi}_{L}^{c}, \bar{\phi}_{L}^{u}, \bar{\phi}_{L}^{v}\right):=\left(\phi_{L}^{\rho}, \phi_{L}^{c}, \phi_{L}^{u}, \phi_{L}^{v}\right)\left(t_{1}, x_{1}, y_{1}\right)-$ $\left(\phi_{L}^{\rho}, \phi_{L}^{c}, \phi_{L}^{u}, \phi_{L}^{v}\right)\left(t_{2}, x_{2}, y_{2}\right)$ and using the fact that $\left(\phi^{u}, \phi^{v}\right) \in S$, one can show that the operator $A$ is equicontinuous.

Equipped with Lemma 5.4, we conclude that the operator $A$ is compact. Hence, by the second Schauder fixed-point theorem, [24], it has at least one fixed point $\left(\rho^{\mathrm{DG}}, c^{\mathrm{DG}}, u^{\mathrm{DG}}, v^{\mathrm{DG}}\right)$, which is the DG solution of (3.3)-(3.6). For this solution, we establish the convergence rate results, stated in the following theorem.

Theorem $5.5\left(L^{2}\left(H^{1}\right)\right.$ - and $L^{\infty}\left(L^{2}\right)$-Error Estimates) Let the solution of the Keller-Segel system (1.3)-(1.6) satisfies the smoothness assumption (5.2). If the penalty parameters $\sigma_{\rho}, \sigma_{c}$, $\sigma_{u}$, and $\sigma_{v}$ in the DG method (3.3)-(3.9) are sufficiently large and $r_{\min } \geq 2$, then there exist constants $C_{\rho}$ and $C_{c}$, independent of $h, r_{\rho}, r_{c}, r_{u}$, and $r_{v}$ such that the following two error estimates hold:

$$
\begin{aligned}
& \left\|\rho^{\mathrm{DG}}-\rho\right\|_{L^{\infty}\left([0, T] ; L^{2}(\Omega)\right)}+\left\|\mid \nabla\left(\rho^{\mathrm{DG}}-\rho\right)\right\| \|_{L^{2}\left([0, T] ; L^{2}(\Omega)\right)}+\left(\int_{0}^{T} \sum_{e \in \Gamma_{h}} \frac{r_{\rho}^{2}}{|e|}\left\|\left[\rho^{\mathrm{DG}}-\rho\right]\right\|_{0, e}^{2}\right)^{\frac{1}{2}} \leq C_{\rho} E, \\
& \left\|c^{\mathrm{DG}}-c\right\|_{L^{\infty}\left([0, T] ; L^{2}(\Omega)\right)}+\|\| \nabla\left(c^{\mathrm{DG}}-c\right)\|\|_{L^{2}\left([0, T] ; L^{2}(\Omega)\right)}+\left(\int_{0}^{T} \sum_{e \in \Gamma_{h}} \frac{r_{c}^{2}}{|e|}\left\|\left[c^{\mathrm{DG}}-c\right]\right\|_{0, e}^{2}\right)^{\frac{1}{2}} \leq C_{c} E,
\end{aligned}
$$

where

$$
E:=\left(\frac{h^{\min \left(r_{\rho}+1, s_{\rho}\right)-1}}{r_{\rho}^{s_{\rho}-2}}+\frac{h^{\min \left(r_{c}+1, s_{c}\right)-1}}{r_{c}^{s_{c}-2}}+\frac{h^{\min \left(r_{u}+1, s_{u}\right)-1}}{r_{u}^{s_{u}-2}}+\frac{h^{\min \left(r_{v}+1, s_{v}\right)-1}}{r_{v}^{s_{v}-2}}\right) .
$$


Proof: The result follows from the definition of space $S$, the fact that the DG solution is a fixed point of the compact operator $A$ (defined above), the $h p$ Approximation Lemma 2.1, and the triangle inequality.

Remark. The obtained error estimates are $h$-optimal, but only suboptimal for $r$.

Finally, equipped with the results established in Theorem 5.5, we obtain the following bound for the blow-up time of the exact solution of the Keller-Segel system.

Theorem 5.6 Let us denote by $t_{b}$ the blow-up time of the exact solution of the Keller-Segel system (1.1) and by $t_{b}^{\mathrm{DG}}$ the blow-up time of the DG solution of (3.3)-(3.9). Then $t_{b} \leq t_{b}^{\mathrm{DG}}$.

Proof: The solution $\rho$ of the Keller-Segel model blows up if $\|\rho\|_{L^{\infty}(\Omega)}$ becomes unbounded in either finite or infinite time (see, e.g., [26, 27]). Therefore, in order to prove the theorem we need to establish an $L^{\infty}$-error bound. Consider interpolant $\widetilde{\rho}$

From Theorem 5.5 we have the following $L^{2}$-error bound:

$$
\left\|\rho^{\mathrm{DG}}-\widetilde{\rho}\right\|_{L^{2}(\Omega)} \leq C_{\rho}\left(\frac{h^{\min \left(r_{\rho}+1, s_{\rho}\right)-1}}{r_{\rho}^{s_{\rho}-2}}+\frac{h^{\min \left(r_{c}+1, s_{c}\right)-1}}{r_{c}^{s_{c}-2}}+\frac{h^{\min \left(r_{u}+1, s_{u}\right)-1}}{r_{u}^{s_{u}-2}}+\frac{h^{\min \left(r_{v}+1, s_{v}\right)-1}}{r_{v}^{s_{v}-2}}\right),
$$

which together with the inverse inequality (2.6) leads to the desired $L^{\infty}$-error bound,

$$
\left\|\rho^{\mathrm{DG}}-\widetilde{\rho}\right\|_{L^{\infty}(\Omega)} \leq C_{\rho}\left(\frac{h^{\min \left(r_{\rho}+1, s_{\rho}\right)-2}}{r_{\rho}^{s_{\rho}-3}}+\frac{h^{\min \left(r_{c}+1, s_{c}\right)-2}}{r_{c}^{s_{c}-3}}+\frac{h^{\min \left(r_{u}+1, s_{u}\right)-2}}{r_{u}^{s_{u}-3}}+\frac{h^{\min \left(r_{v}+1, s_{v}\right)-2}}{r_{v}^{s_{v}-3}}\right),
$$

which, in turn, implies that

$\left\|\rho^{\mathrm{DG}}\right\|_{L^{\infty}(\Omega)} \leq\|\widetilde{\rho}\|_{L^{\infty}(\Omega)}+C_{\rho}\left(\frac{h^{\min \left(r_{\rho}+1, s_{\rho}\right)-2}}{r_{\rho}^{s_{\rho}-3}}+\frac{h^{\min \left(r_{c}+1, s_{c}\right)-2}}{r_{c}^{s_{c}-3}}+\frac{h^{\min \left(r_{u}+1, s_{u}\right)-2}}{r_{u}^{s_{u}-3}}+\frac{h^{\min \left(r_{v}+1, s_{v}\right)-2}}{r_{v}^{s_{v}-3}}\right)$.

From the last estimate the statement of the theorem follows.

\section{$6 \quad$ Numerical Example}

In this section, we demonstrate the performance of the proposed DG method. In all our numerical experiments, we have used the third-order strong stability preserving Runge-Kutta method for the time discretization, [23]. No slope limiting technique has been implemented. The values of the penalty parameters used are $\sigma_{\rho}=\sigma_{c}=1$ and $\sigma_{u}=\sigma_{v}=0.01$. We note that no instabilities have been observed when the latter two parameters were taken zero, however, since our convergence proof requires $\sigma_{u}$ and $\sigma_{v}$ to be positive, we only show the results obtained with positive $\sigma_{u}$ and $\sigma_{v}$, which are almost identical to the ones obtained with $\sigma_{u}=\sigma_{v}=0$.

We consider the initial-boundary value problem for the Keller-Segel system in the square domain $\left[-\frac{1}{2}, \frac{1}{2}\right] \times\left[-\frac{1}{2}, \frac{1}{2}\right]$. We take the chemotactic sensitivity $\chi=1$ and the bell-shaped initial data

$$
\rho(x, y, 0)=1200 e^{-120\left(x^{2}+y^{2}\right)}, \quad c(x, y, 0)=600 e^{-60\left(x^{2}+y^{2}\right)} .
$$

According to the results in [25], both components $\rho$ and $c$ of the solution are expected to blow up at the origin in finite time. This situation is especially challenging since capturing blowing up solution with shrinking support is extremely hard. 
In Figures 6.1-6.4, we plot the logarithmically scaled density, $\ln \left(1+\rho^{\mathrm{DG}}\right)$, computed at different times on two different uniform grids with $h=1 / 51$ (Figures 6.1 and 6.3) and $h=1 / 101$ (Figures 6.2 and 6.4). The results shown in Figures 6.1-6.2 have been obtained with quadratic polynomials (i.e., $r_{\rho}=r_{c}=r_{u}=r_{v}=r=2$ ), while the solution shown in Figures 6.3-6.4 have been computed with the help of cubic polynomials (i.e., $r_{\rho}=r_{c}=r_{u}=r_{v}=r=3$ ).

Numerical convergence of the scheme is verified by refining the mesh and by increasing the polynomial degree. As one can see, the computed solutions in a very good agreement at the smaller times $\left(t=1.46 \cdot 10^{-5}, 2.99 \cdot 10^{-5}\right.$, and $\left.6.03 \cdot 10^{-5}\right)$. However, at time close to the blowup time $\left(t=1.21 \cdot 10^{-4}\right)$ the maximum value of $\rho^{\mathrm{DG}}$ grows while its support shrinks, and no mesh-refinement convergence is observed: the numerical solution keeps increasing when the mesh is refined. Using Theorem 5.6, we can conclude that in this example, the blow-up time of the exact solution is less or equal to the blow-up time of the DG solution, which is approximately $t_{b}^{\mathrm{DG}} \approx 1.21 \cdot 10^{-4}$.

We note that even though no slope limiting or any other positivity preserving techniques have been implemented, the computed solutions have never developed negative values and are oscillation-free.
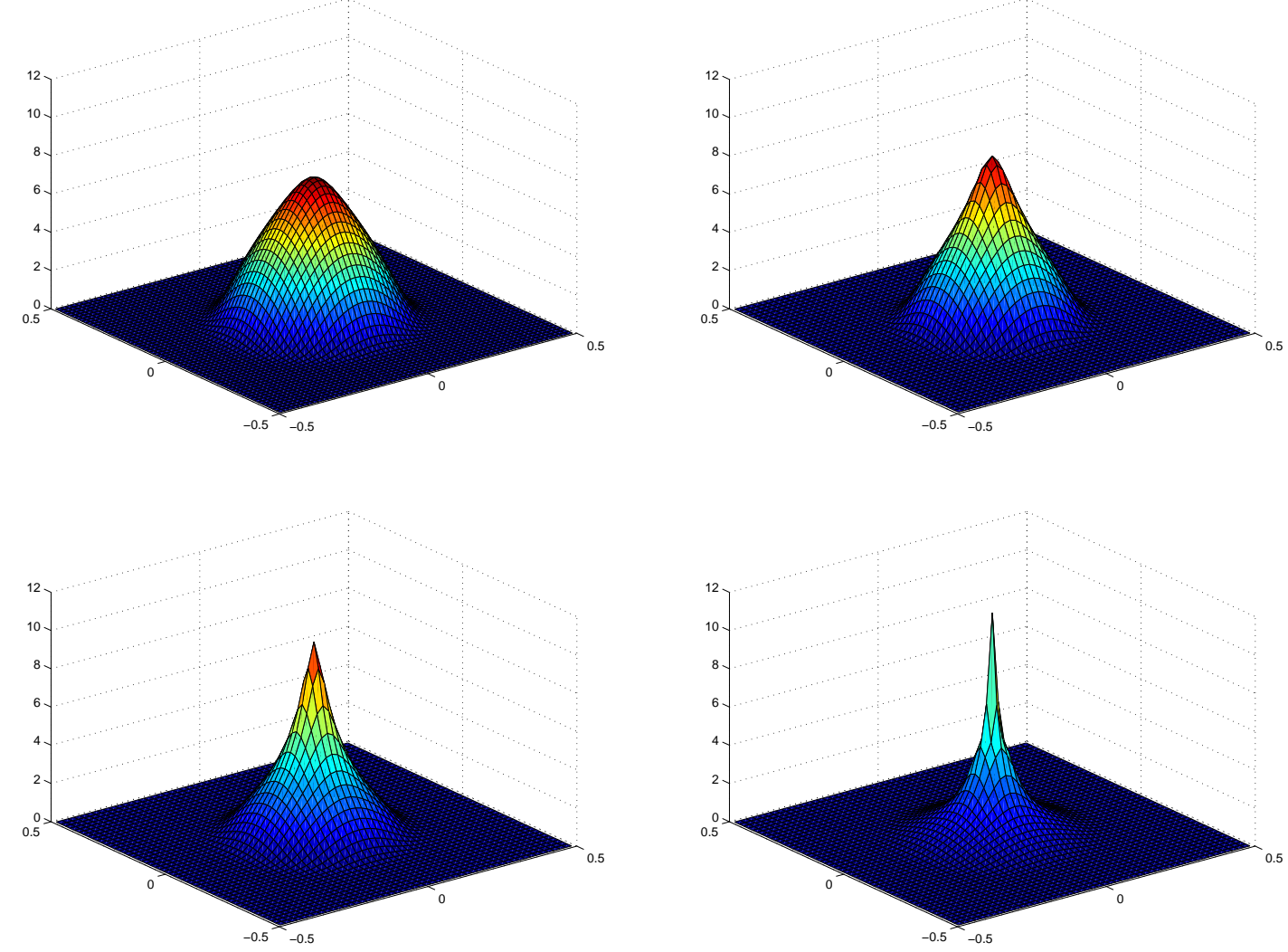

Figure 6.1: $h=1 / 51, r=2$. Logarithmically scaled density computed at $t=1.46 \cdot 10^{-5}$ (top left), $t=2.99 \cdot 10^{-5}$ (top right), $t=6.03 \cdot 10^{-5}$ (bottom left), and $t=1.21 \cdot 10^{-4} \approx t_{b}^{\mathrm{DG}}$ (bottom right).

Finally, we check the numerical order of the convergence of the proposed DG method. We first consider the smooth solution at a very small time $t=1.0 \cdot 10^{-7}$ and test the convergence with 

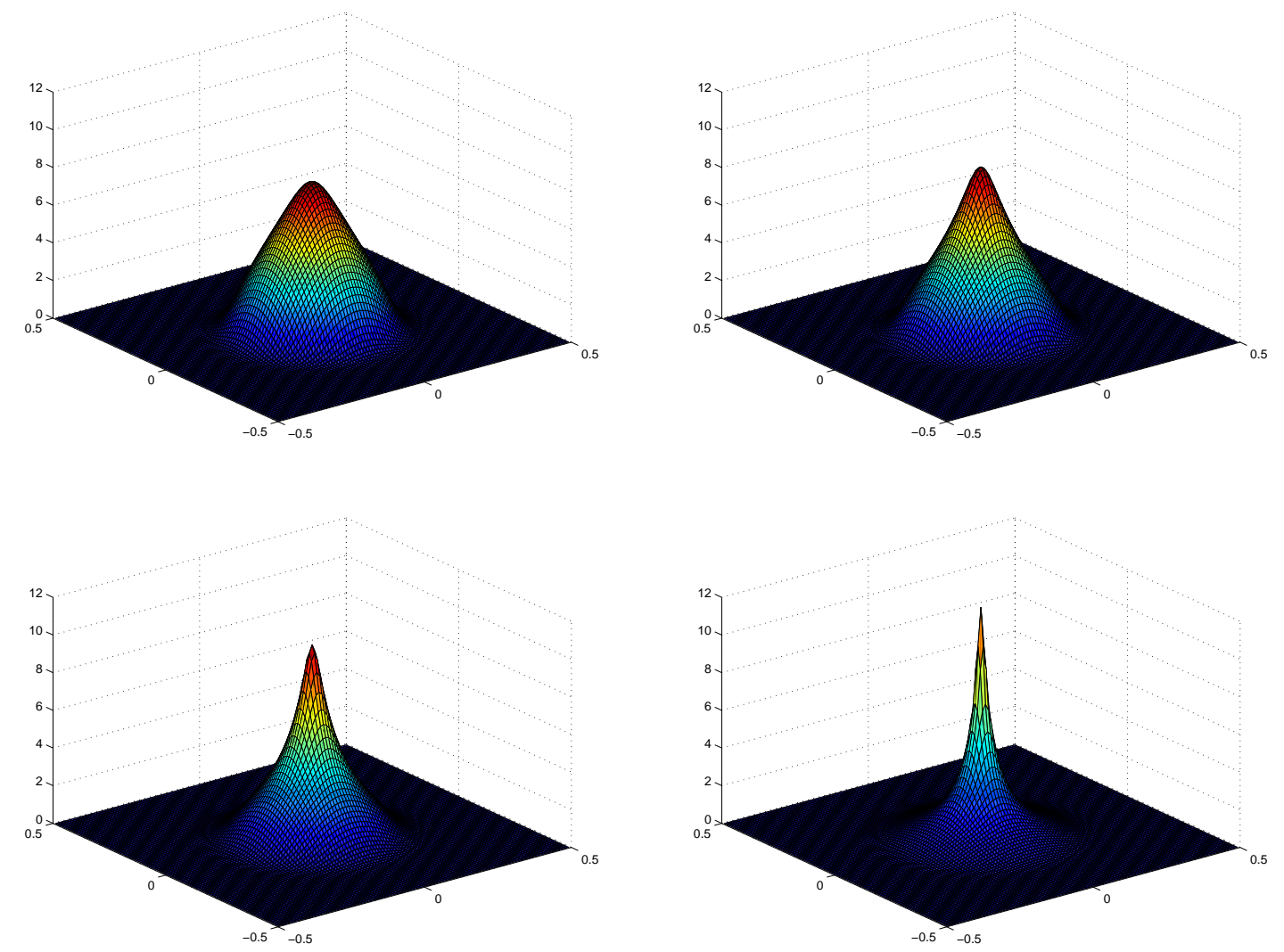

Figure 6.2: The same as in Figure 6.1 but with $h=1 / 101, r=2$.

respect to the mesh size $h$ for the fixed $r=2$ (piecewise quadratic polynomials). Since the exact solution for the Keller-Segel system is unavailable, we compute the reference solution by the proposed DG method on a fine mesh with $h=1 / 128$ and using the fifth-order $(r=5)$ piecewise polynomials. We then use the obtained reference solution to compute the relative $L^{2}$ - and relative $H^{1}$-errors. These errors are presented in Table 6.1. From this table, one can see that the solution numerically converges to the reference solution with the (optimal) second order in the $H^{1}$-norm which confirms the theoretical results predicted by our convergence analysis. Moreover, the achieved third order of convergence in the $L^{2}$-norm is optimal for quadratic piecewise polynomials.

We then test the convergence of the proposed DG method with respect to the degree $r$ of piecewise polynomials for the fixed $h=1 / 32$. The obtained results, reported in Table 6.2 , show that the error decreases almost exponentially when the polynomial degree increases (this is a typical situation when DG methods capture smooth solutions).

We also compute the $L^{2}$-errors with respect to the reference solution, for the solutions plotted on Figures 6.1 and 6.2 at times $t=2.99 \cdot 10^{-5}$ and $t=6.03 \cdot 10^{-5}$. These times are close to the blowup time and the solutions develop a pick at the origin. The obtained errors are reported in Table 6.3. As one can see, even for the spiky solutions, the convergence rate is very high though it, as expected, deteriorates as $t$ approaches $t_{b}^{\mathrm{DG}}$. 

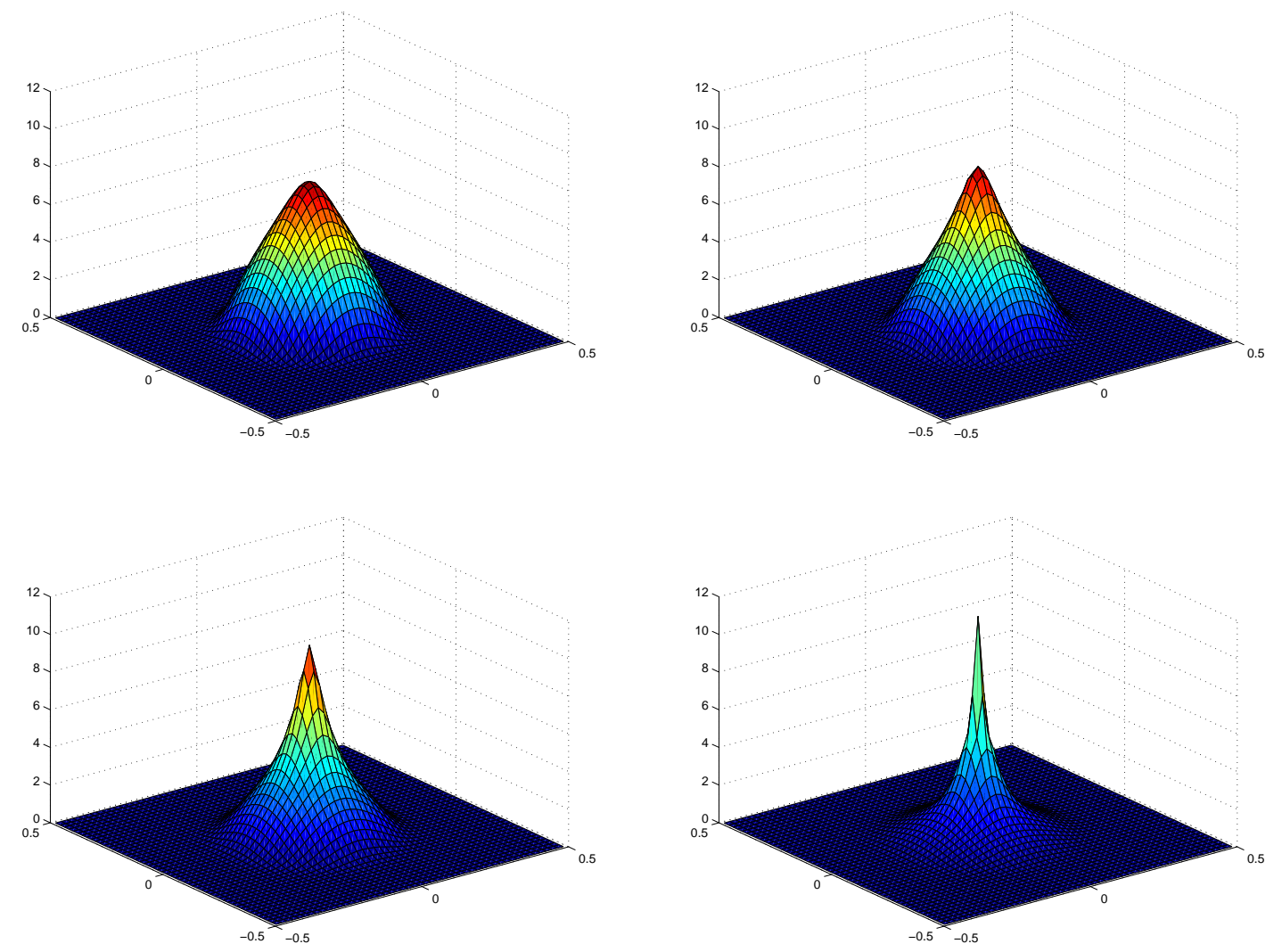

Figure 6.3: The same as in Figures $6.1-6.2$ but with $h=1 / 51, r=3$.

\begin{tabular}{|c|rc|rc|}
\hline$h$ & $L^{2}$-error & Rate & $H^{1}$-error & Rate \\
\hline $1 / 4$ & 3.0578 & - & 1.5591 & - \\
$1 / 8$ & 1.0290 & 1.6 & 1.2348 & 0.35 \\
$1 / 16$ & 0.0796 & 3.7 & 0.5206 & 1.3 \\
$1 / 32$ & 0.0075 & 3.4 & 0.0937 & 2.5 \\
$1 / 64$ & 0.0006 & 3.6 & 0.0157 & 2.6 \\
\hline
\end{tabular}

Table 6.1: Relative errors as functions of the mesh size $h ; r=2$ is fixed.

\begin{tabular}{|c|rc|rc|}
\hline$r$ & $L^{2}$-error & Rate & $H^{1}$-error & Rate \\
\hline 2 & $7.5 \mathrm{e}-03$ & - & $9.4 \mathrm{e}-02$ & - \\
3 & $9.0 \mathrm{e}-04$ & 5.2 & $2.2 \mathrm{e}-02$ & 3.6 \\
4 & $8.0 \mathrm{e}-05$ & 8.4 & $2.6 \mathrm{e}-03$ & 7.4 \\
5 & $6.9 \mathrm{e}-06$ & 11.0 & $2.9 \mathrm{e}-04$ & 9.8 \\
\hline
\end{tabular}

Table 6.2: Relative errors as functions of the piecewise polynomial degree $r ; h=1 / 32$ is fixed. 

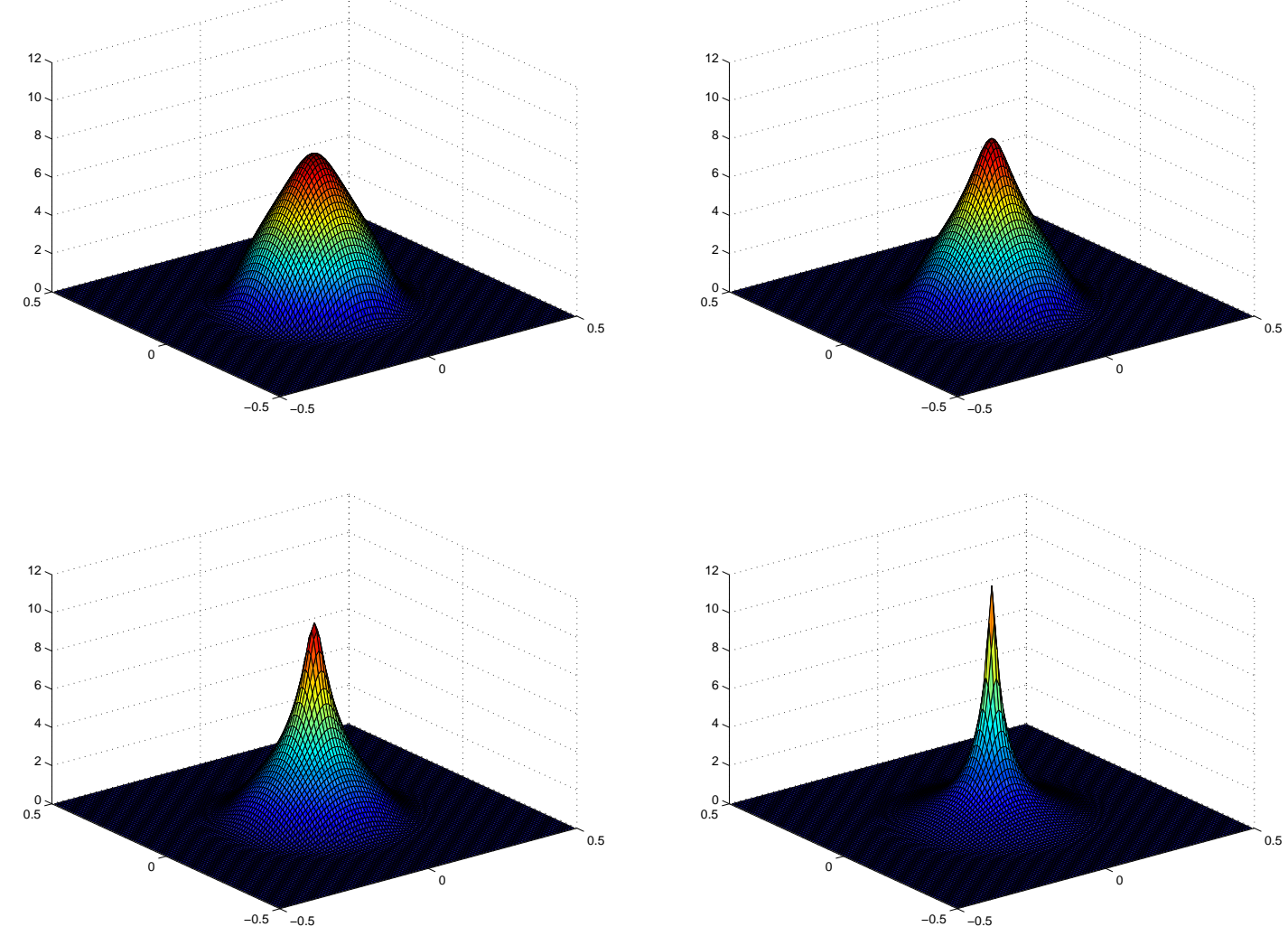

Figure 6.4: The same as in Figures 6.1-6.3 but with $h=1 / 101, r=3$.

\section{Appendix A: Proof of Theorem 5.3 - Continuation}

In this appendix, we complete the proof of Theorem 5.3 by proving that $\left(\phi_{L}^{c}, \phi_{L}^{u}, \phi_{L}^{v}\right) \in S$.

We begin with $\phi_{L}^{c}$ and show that $\phi_{L}^{c} \in S$ in a way similar to the proof of the fact that $\phi_{L}^{\rho} \in S$ given in $\S 5$. First, from the consistency Lemma 4.1 we obtain that the exact solution of (1.3)-(1.6) satisfies equation (3.4), which may be rewritten as

$$
\begin{aligned}
& \int_{\Omega} \widetilde{c}_{t} w^{c}+\sum_{E \in \mathcal{E}_{h}} \int_{E} \nabla \widetilde{c} \nabla w^{c}-\sum_{e \in \Gamma_{h}} \int_{e}\left\{\nabla \widetilde{c} \cdot \mathbf{n}_{e}\right\}\left[w^{c}\right]+\varepsilon \sum_{e \in \Gamma_{h}} \int_{e}\left\{\nabla w^{c} \cdot \mathbf{n}_{e}\right\}[\widetilde{c}]+\sigma_{c} \sum_{e \in \Gamma_{h}} \frac{r_{c}^{2}}{|e|} \int_{e}[\widetilde{c}]\left[w^{c}\right] \\
& \quad+\int_{\Omega} \widetilde{c} w^{c}-\int_{\Omega} \widetilde{\rho} w^{c}=-\int_{\Omega} \xi_{t}^{c} w^{c}-\sum_{E \in \mathcal{E}_{h}} \int_{E} \nabla \xi^{c} \nabla w^{c}+\sum_{e \in \Gamma_{h}} \int_{e}\left\{\nabla \xi^{c} \cdot \mathbf{n}_{e}\right\}\left[w^{c}\right] \\
& -\varepsilon \sum_{e \in \Gamma_{h}} \int_{e}\left\{\nabla w^{c} \cdot \mathbf{n}_{e}\right\}\left[\xi^{c}\right]-\sigma_{c} \sum_{e \in \Gamma_{h}} \frac{r_{c}^{2}}{|e|} \int_{e}\left[\xi^{c}\right]\left[w^{c}\right]-\int_{\Omega} \xi^{c} w^{c}+\int_{\Omega} \xi^{\rho} w^{c} .
\end{aligned}
$$

We then subtract equation (A.1) from equation (5.5) and set $w^{c}=\tau^{c}$ to obtain

$$
\frac{1}{2} \frac{d}{d t}\left\|\tau^{c}\right\|_{0, \Omega}^{2}+\left\|\tau^{c}\right\|_{0, \Omega}^{2}+\|\| \nabla \tau^{c}\left\|_{0, \Omega}^{2}+\sigma_{c} \sum_{e \in \Gamma_{h}} \frac{r_{c}^{2}}{|e|}\right\|\left[\tau^{c}\right] \|_{0, e}^{2}
$$




\begin{tabular}{|c|cc|cc|}
\hline \multirow{2}{*}{$h$} & \multicolumn{2}{|c|}{$t=2.99 \cdot 10^{-5}$} & \multicolumn{2}{|c|}{$t=6.03 \cdot 10^{-5}$} \\
\cline { 2 - 5 } & $L^{2}$-error & Rate & $L^{2}$-error & Rate \\
\hline $1 / 51$ & $5.5 \mathrm{e}-02$ & - & $5.0 \mathrm{e}-02$ & - \\
$1 / 101$ & $5.2 \mathrm{e}-03$ & 3.4 & $1.1 \mathrm{e}-02$ & 2.2 \\
\hline
\end{tabular}

Table 6.3: Relative $L^{2}$-errors at two different times; $r=2$ is fixed.

$$
\begin{aligned}
& =\int_{\Omega} \tau^{\rho} \tau^{c}+(1-\varepsilon) \sum_{e \in \Gamma_{h}} \int_{e}\left\{\nabla \tau^{c} \cdot \mathbf{n}_{e}\right\}\left[\tau^{c}\right]+\int_{\Omega} \xi_{t}^{c} \tau^{c}+\sum_{E \in \mathcal{E}_{h}} \int_{E} \nabla \xi^{c} \nabla \tau^{c}-\sum_{e \in \Gamma_{h}} \int_{e}\left\{\nabla \xi^{c} \cdot \mathbf{n}_{e}\right\}\left[\tau^{c}\right] \\
& +\varepsilon \sum_{e \in \Gamma_{h}} \int_{e}\left\{\nabla \tau^{c} \cdot \mathbf{n}_{e}\right\}\left[\xi^{c}\right]+\sigma_{c} \sum_{e \in \Gamma_{h}} \frac{r_{c}^{2}}{|e|} \int\left[\xi_{e}^{c}\right]\left[\tau^{c}\right]+\int_{\Omega} \xi^{c} \tau^{c}-\int_{\Omega} \xi^{\rho} \tau^{c}=: T_{1}^{c}+\ldots+T_{9}^{c} .
\end{aligned}
$$

Next, we bound each term on the RHS of (A.2).

We begin with the term $T_{1}^{c}$. We first bound it using the Cauchy-Schwarz and Young's inequalities, and then apply the estimate (5.33). This results in

$$
\begin{aligned}
& \left|T_{1}^{c}\right| \leq\left\|\tau^{c}\right\|_{0, \Omega}^{2}+\frac{1}{4}\left\|\tau^{\rho}\right\|_{0, \Omega}^{2} \\
& \leq\left\|\tau^{c}\right\|_{0, \Omega}^{2}+\frac{C^{\mathrm{I}}}{4}\left(\frac{h^{2 \min \left(r_{\rho}+1, s_{\rho}\right)-2}}{r_{\rho}^{2 s_{\rho}-4}}+\frac{h^{2 \min \left(r_{c}+1, s_{c}\right)-2}}{r_{c}^{2 s_{c}-4}}+\frac{h^{2 \min \left(r_{u}+1, s_{u}\right)-2}}{r_{u}^{2 s_{u}-4}}+\frac{h^{2 \min \left(r_{v}+1, s_{v}\right)-2}}{r_{v}^{2 s_{v}-4}}\right) .
\end{aligned}
$$

The terms $T_{2}^{c}, T_{3}^{c}, T_{4}^{c}, T_{5}^{c}, T_{6}^{c}$, and $T_{7}^{c}$ are similar to the terms $T_{1}^{\rho}, T_{6}^{\rho}, T_{7}^{\rho}, T_{8}^{\rho}, T_{9}^{\rho}$, and $T_{10}^{\rho}$ estimated in (5.17), (5.23), (5.24), (5.25), (5.26), and (5.27), respectively. Hence, they can be bounded as follows:

$$
\begin{aligned}
& \left|T_{2}^{c}\right| \leq \varepsilon_{2}^{c}\left|\left\|\nabla \tau^{c} \mid\right\|_{0, \Omega}^{2}+C_{2}^{c} \sum_{e \in \Gamma_{h}} \frac{r_{c}^{2}}{|e|}\left\|\left[\tau^{c}\right]\right\|_{0, e}^{2},\right. \\
& \left|T_{3}^{c}\right| \leq\left\|\tau^{c}\right\|_{0, \Omega}^{2}+C^{*} \frac{h^{2 \min \left(r_{c}+1, s_{c}\right)}}{r_{c}^{2 s_{c}}}, \\
& \left|T_{4}^{c}\right| \leq \varepsilon_{4}^{c}\left|\left\|\nabla \tau^{c} \mid\right\|_{0, \Omega}^{2}+C^{*} \frac{h^{2 \min \left(r_{c}+1, s_{c}\right)-2}}{r_{c}^{2 s_{c}-2}},\right. \\
& \left|T_{5}^{c}\right| \leq C_{5}^{c} \sum_{e \in \Gamma_{h}} \frac{r_{c}^{2}}{|e|}\left\|\left[\tau^{c}\right]\right\|_{0, e}^{2}+C^{*} \frac{h^{2 \min \left(r_{c}+1, s_{c}\right)-2}}{r_{c}^{2 s_{c}-2}}, \\
& \left|T_{6}^{c}\right| \leq \varepsilon_{6}^{c}\left|\| \nabla \tau^{c}\right|||_{0, \Omega}^{2}+C^{*} \frac{h^{2 \min \left(r_{c}+1, s_{c}\right)-2}}{r_{c}^{2 s_{c}-4}}, \\
& \left|T_{7}^{c}\right| \leq C_{6}^{c} \sum_{e \in \Gamma_{h}} \frac{\tau_{c}^{2}}{|e|}\left\|\left[\tau^{c}\right]\right\|_{0, e}^{2}+C^{*} \frac{h^{2 \min \left(r_{c}+1, s_{c}\right)-2}}{r_{c}^{2 s_{c}-4}} .
\end{aligned}
$$

Finally, the last two terms on the RHS of (A.2), $T_{8}^{c}$ and $T_{9}^{c}$, are bounded using the CauchySchwarz inequality, Young's inequality, and the approximation inequality (2.2):

$$
\left|T_{8}^{c}\right| \leq\left\|\tau^{c}\right\|_{0, \Omega}^{2}+C^{*} \frac{h^{2 \min \left(r_{c}+1, s_{c}\right)}}{r_{c}^{2 s_{c}}}, \quad\left|T_{9}^{c}\right| \leq\left\|\tau^{c}\right\|_{0, \Omega}^{2}+C^{*} \frac{h^{2 \min \left(r_{\rho}+1, s_{\rho}\right)}}{r_{\rho}^{2 s_{\rho}}} .
$$


Now substituting (A.3)-(A.10) into (A.2) and using the assumption that $h<1$, we obtain the following estimate for $\tau^{c}$ :

$$
\begin{aligned}
& \frac{1}{2} \frac{d}{d t}\left\|\tau^{c}\right\|_{0, \Omega}^{2}+\left(1-\varepsilon_{2}^{c}-\varepsilon_{4}^{c}-\varepsilon_{6}^{c}\right)\|\| \nabla \tau^{c}\left\|\left.\right|_{0, \Omega} ^{2}+\left(\sigma_{c}-C_{2}^{c}-C_{5}^{c}-C_{6}^{c}\right) \sum_{e \in \Gamma_{h}} \frac{r_{c}^{2}}{|e|}\right\|\left[\tau^{c}\right] \|_{0, e}^{2} \\
& \leq 3\left\|\tau^{c}\right\|_{0, \Omega}^{2}+C_{*}^{c}\left(\frac{h^{2 \min \left(r_{\rho}+1, s_{\rho}\right)-2}}{r_{\rho}^{2 s_{\rho}-4}}+\frac{h^{2 \min \left(r_{c}+1, s_{c}\right)-2}}{r_{c}^{2 s_{c}-4}}+\frac{h^{2 \min \left(r_{u}+1, s_{u}\right)-2}}{r_{u}^{2 s_{u}-4}}+\frac{h^{2 \min \left(r_{v}+1, s_{v}\right)-2}}{r_{v}^{2 s_{v}-4}}\right) .
\end{aligned}
$$

This estimate is similar to the estimate (5.31). After a proper selection of $\varepsilon_{i}^{c}$ and the penalty parameter $\sigma_{c}$, we multiply (A.11) by 2, integrate with respect to time from 0 to $t$, apply Gronwall's Lemma 2.6, and take the supremum over $t$. This results in an estimate, which is completely analogous to (5.33):

$$
\begin{aligned}
& \sup _{[0, T]}\left\|\tau^{c}\right\|_{0, \Omega}^{2}+\int_{0}^{T}\left(\left\|\nabla \tau^{c} \mid\right\|_{0, \Omega}^{2}+\sum_{e \in \Gamma_{h}} \frac{r_{c}^{2}}{|e|}\left\|\left[\tau^{c}\right]\right\|_{0, e}^{2}\right) \\
& \leq C^{\mathrm{II}}\left(\frac{h^{2 \min \left(r_{\rho}+1, s_{\rho}\right)-2}}{r_{\rho}^{2 s_{\rho}-4}}+\frac{h^{2 \min \left(r_{c}+1, s_{c}\right)-2}}{r_{c}^{2 s_{c}-4}}+\frac{h^{2 \min \left(r_{u}+1, s_{u}\right)-2}}{r_{u}^{2 s_{u}-4}}+\frac{h^{2 \min \left(r_{v}+1, s_{v}\right)-2}}{r_{v}^{2 s_{v}-4}}\right)
\end{aligned}
$$

where $C^{\mathrm{II}}$ is a constant that depends on $\|\rho\|_{\left(L^{\infty}([0, T]) ; H^{2}(\Omega)\right)},\left\|\rho_{t}\right\|_{\left(L^{\infty}([0, T]) ; L^{2}(\Omega)\right)},\|c\|_{\left(L^{\infty}([0, T]) ; H^{2}(\Omega)\right)}$, $\left\|c_{t}\right\|_{\left(L^{\infty}([0, T]) ; L^{2}(\Omega)\right)},\|u\|_{\left(L^{\infty}([0, T]) ; L^{2}(\Omega)\right)},\|v\|_{\left(L^{\infty}([0, T]) ; L^{2}(\Omega)\right)}$, and $T$ only.

Hence, according to the definition on page 9, the estimate (A.12) implies that $\phi_{L}^{c} \in S$.

Next, we proceed with proving that $\phi_{L}^{u} \in S$. Once again, by the consistency Lemma (4.1), the exact solution satisfies the following equation (compare it with (3.5)):

$$
\begin{gathered}
\int_{\Omega} \tilde{u} w^{u}+\sum_{E \in \mathcal{E}_{h}} \int_{E} \widetilde{c}\left(w^{u}\right)_{x}+\sum_{e \in \Gamma_{h}^{\mathrm{ver}}} \int_{e}(-c)_{u}^{* *} n_{x}\left[w^{u}\right]-\sum_{e \in \partial \Omega_{\mathrm{ver}}} \int_{e} \widetilde{c} n_{x} w^{u}+\sigma_{u} \sum_{e \in \Gamma_{h} \cup \partial \Omega_{\mathrm{ver}}} \frac{r_{u}^{2}}{|e|} \int_{e}[\widetilde{u}]\left[w^{u}\right] \\
=-\int_{\Omega} \xi^{u} w^{u}-\sum_{E \in \mathcal{E}_{h}} \int_{E} \xi^{c}\left(w^{u}\right)_{x}+\sum_{e \in \partial \Omega_{\mathrm{ver}}} \int_{e} \xi^{c} n_{x} w^{u}-\sigma_{u} \sum_{e \in \Gamma_{h} \cup \partial \Omega_{\mathrm{ver}}} \frac{r_{u}^{2}}{|e|} \int_{e}\left[\xi^{u}\right]\left[w^{u}\right],
\end{gathered}
$$

where

$$
(-c)_{u}^{* *}:=-\frac{a_{L}^{\text {out }} c_{e}^{E^{1}}-a_{L}^{\text {in }} c_{e}^{E^{2}}}{a_{L}^{\text {out }}-a_{L}^{\text {in }}}-\frac{a_{L}^{\text {out }} a_{L}^{\text {in }}}{a_{L}^{\text {out }}-a_{L}^{\text {in }}}[u] .
$$

Subtracting equation (A.13) from (5.6) and choosing $w^{u}=\tau^{u}$, we obtain

$$
\begin{aligned}
& \left\|\tau^{u}\right\|_{0, \Omega}^{2}+\sigma_{u} \sum_{e \in \Gamma_{h} \cup \partial \Omega_{\mathrm{ver}}} \frac{r_{u}^{2}}{|e|}\left\|\left[\tau^{u}\right]\right\|_{0, \Omega}^{2} \\
& =-\sum_{E \in \mathcal{E}_{h}} \int_{E} \tau^{c}\left(\tau^{u}\right)_{x}-\sum_{e \in \Gamma_{h}^{\mathrm{ver}}} \int_{e}\left(\left(-\phi_{L}^{c}\right)_{u}^{*}-(-c)_{u}^{* *}\right) n_{x}\left[\tau^{u}\right]+\sum_{e \in \partial \Omega_{\mathrm{ver}}} \int_{e} \tau^{c} n_{x}\left[\tau^{u}\right]+\int_{\Omega} \xi^{u} \tau^{u} \\
& \quad+\sum_{E \in \mathcal{E}_{h}} \int_{E} \xi^{c}\left(\tau^{u}\right)_{x}-\sum_{e \in \partial \Omega_{\mathrm{ver}}} \int_{e} \xi^{c} n_{x}\left[\tau^{u}\right]+\sigma_{u} \sum_{e \in \Gamma_{h} \cup \partial \Omega_{\mathrm{ver}}} \frac{r_{u}^{2}}{|e|} \int_{e}\left[\xi^{u}\right]\left[\tau^{u}\right]=: T_{1}^{u}+\ldots+T_{7}^{u},(\mathrm{~A} .
\end{aligned}
$$


and bound each term on the RHS of (A.14).

To estimate the term $T_{1}^{u}$, we first integrate by parts and rewrite it as

$$
T_{1}^{u}=-\left(-\sum_{E \in \mathcal{E}_{h}} \int_{E}\left(\tau^{c}\right)_{x} \tau^{u}+\sum_{E \in \mathcal{E}_{h}} \sum_{e \in \partial E} \int_{e} \tau^{c} \tau^{u} n_{x}\right)=\sum_{E \in \mathcal{E}_{h}} \int_{E}\left(\tau^{c}\right)_{x} \tau^{u}-\sum_{e \in \Gamma_{h}^{\text {ver }} \cup \partial \Omega_{\mathrm{ver}}} \int_{e}\left[\tau^{c} \tau^{u}\right] n_{x} .
$$

Then, using the formula for the jump and the average operators (2.1), we obtain

$$
T_{1}^{u}=\sum_{E \in \mathcal{E}_{h}} \int_{E}\left(\tau^{c}\right)_{x} \tau^{u}-\sum_{e \in \Gamma_{h}^{\mathrm{ver}}} \int_{e}\left[\tau^{c}\right]\left\{\tau^{u}\right\} n_{x}-\sum_{e \in \Gamma_{h}^{\mathrm{ver}}} \int_{e}\left[\tau^{u}\right]\left\{\tau^{c}\right\} n_{x}-\sum_{e \in \partial \Omega_{\mathrm{ver}}} \int_{e} \tau^{c}\left[\tau^{u}\right] n_{x} .
$$

Hence, using the Cauchy-Schwarz inequality, Young's inequality, the inequality (2.5), and applying the assumption (3.2), we arrive at the following bound for $T_{1}^{u}$ :

$$
\left|T_{1}^{u}\right| \leq \frac{9}{16}\left\|\tau^{u}\right\|_{0, \Omega}^{2}+C_{1}^{u} \sum_{e \in \Gamma_{h} \cup \partial \Omega_{\mathrm{ver}}} \frac{r_{u}^{2}}{|e|}\left\|\left[\tau^{u}\right]\right\|_{0, e}^{2}+\frac{1}{2}\left\|\left(\tau^{c}\right)_{x}\right\|\left\|_{0, \Omega}^{2}+C_{2}^{u}\right\| \tau^{c}\left\|_{0, \Omega}^{2}+C_{3}^{u} \sum_{e \in \Gamma_{h}} \frac{r_{c}^{2}}{|e|}\right\|\left[\tau^{c}\right] \|_{0, e}^{2} .
$$

A bound for $T_{2}^{u}$ can be obtained in a way similar to the one the bound on $T_{3}^{\rho}$ has been established:

$$
\begin{aligned}
\left|T_{2}^{u}\right| & \leq \sum_{e \in \Gamma_{h}^{\text {ver }}}\left(\left|\int_{e} \frac{a_{L}^{\text {out }}}{a_{L}^{\text {out }}-a_{L}^{\text {in }}}\left(\left(\phi_{L}^{c}\right)_{e}^{E^{1}}-c_{e}^{E^{1}}\right) n_{x}\left[\tau^{u}\right]\right|+\left|\int_{e} \frac{-a_{L}^{\text {in }}}{a_{L}^{\text {out }}-a_{L}^{\text {in }}}\left(\left(\phi_{L}^{c}\right)_{e}^{E^{2}}-c_{e}^{E^{2}}\right) n_{x}\left[\tau^{u}\right]\right|\right. \\
& \left.+\left|\int_{e} \frac{-a_{L}^{\text {in }} a_{L}^{\text {out }}}{a_{L}^{\text {out }}-a_{L}^{\text {in }}}\left[\phi_{L}^{u}-u\right] n_{x}\left[\tau^{u}\right]\right|\right):=\mathrm{I}+\mathrm{II}+\text { III. }
\end{aligned}
$$

From (5.10) and (5.13), the first term on the RHS of (A.16) can be estimated by

$$
\mathrm{I} \leq \sum_{e \in \Gamma_{h}}\left(\left|\int_{e}\left(\tau^{c}\right)_{e}^{E^{1}} n_{x}\left[\tau^{u}\right]\right|+\left|\int_{e}\left(\xi^{c}\right)_{e}^{E^{1}} n_{x}\left[\tau^{u}\right]\right|\right):=\widetilde{\mathrm{I}}
$$

Using then the Cauchy-Schwarz inequality, the trace inequality (2.3), the inequality (2.5), and the assumption (3.2), we estimate $\widetilde{I}$ as follows:

$$
\widetilde{\mathrm{I}} \leq \frac{1}{2}\left\|\tau^{c}\right\|_{0, \Omega}^{2}+K \sum_{e \in \Gamma_{h}} \frac{r_{u}^{2}}{|e|}\left\|\left[\tau^{u}\right]\right\|_{0, e}^{2}+C \frac{h^{2 \min \left(r_{c}+1, s_{c}\right)}}{r_{c}^{2 s_{c}}} .
$$

A similar bound can be derived for the second term on the RHS of (A.16). The third term on the RHS of (A.16) is similar to the third term on the RHS of (5.19), hence it can be bounded by

$$
\mathrm{III} \leq\left(\frac{K_{1} h}{r_{u}^{2}}+K_{2}\right) \sum_{e \in \Gamma_{h}} \frac{r_{u}^{2}}{|e|}\left\|\left[\tau^{u}\right]\right\|_{0, e}^{2}+C \frac{h^{2 \min \left(r_{u}+1, s_{u}\right)}}{r_{u}^{2 s_{u}}} .
$$

Combining the above bounds on I, II, and III, we arrive at

$$
\left|T_{2}^{u}\right| \leq\left\|\tau^{c}\right\|_{0, \Omega}^{2}+C_{4}^{u} \sum_{e \in \Gamma_{h}} \frac{r_{u}^{2}}{|e|}\left\|\left[\tau^{u}\right]\right\|_{0, e}^{2}+C^{*}\left(\frac{h^{2 \min \left(r_{u}+1, s_{u}\right)}}{r_{u}^{2 s_{u}}}+\frac{h^{2 \min \left(r_{c}+1, s_{c}\right)}}{r_{c}^{2 s_{c}}}\right) .
$$


To bound the term $T_{3}^{u}$, we use the Cauchy-Schwarz inequality, Young's inequality, and the inequality (2.5), which yield

$$
\left|T_{3}^{u}\right| \leq C_{5}^{u}\left\|\tau^{c}\right\|_{0, \Omega}^{2}+C_{6}^{u} \sum_{e \in \partial \Omega_{\mathrm{ver}}} \frac{r_{u}^{2}}{|e|}\left\|\left[\tau^{u}\right]\right\|_{0, e}^{2} .
$$

The term $T_{4}^{u}$ is bounded with the help of Cauchy-Schwarz inequality, Young's inequality, and the approximation inequality (2.2):

$$
\left|T_{4}^{u}\right| \leq \frac{1}{16}\left\|\tau^{u}\right\|_{0, \Omega}^{2}+C^{*} \frac{h^{2 \min \left(r_{u}+1, s_{u}\right)}}{r_{u}^{2 s_{u}}} .
$$

Using the Cauchy-Schwarz inequality and the inverse inequality (2.7), we first bound $T_{5}^{u}$ by

$$
\left|T_{5}^{u}\right| \leq \sum_{E \in \mathcal{E}_{h}}\left\|\xi^{c}\right\|_{0, E}\left\|\left(\tau^{u}\right)_{x}\right\|_{0, E} \leq \sum_{E \in \mathcal{E}_{h}}\left\|\xi^{c}\right\|_{0, E} h^{-1} r_{u}^{2}\left\|\tau^{u}\right\|_{0, E}:=\widetilde{T}_{5}^{u}
$$

We then use Young's inequality, the assumption (3.2), and the approximation inequality (2.2) to obtain

$$
\widetilde{T}_{5}^{u} \leq \frac{1}{16}\left\|\tau^{u}\right\|_{0, \Omega}^{2}+C^{*} \frac{h^{2 \min \left(r_{c}+1, s_{c}\right)-2}}{r_{c}^{2 s_{c}-4}}
$$

The term $T_{6}^{u}$ is bounded using the Cauchy-Schwarz inequality, the trace inequality (2.3), and the approximation inequality (2.2):

$$
\left|T_{6}^{u}\right| \leq C_{7}^{u} \sum_{e \in \partial \Omega_{\mathrm{ver}}} \frac{r_{u}^{2}}{|e|}\left\|\left[\tau^{u}\right]\right\|_{0, e}^{2}+C^{*} \frac{h^{2 \min \left(r_{c}+1, s_{c}\right)}}{r_{c}^{2 s_{c}}} .
$$

The last term $T_{7}^{u}$ is similar to term $T_{10}^{\rho}$, estimated in (5.27). Hence,

$$
\left|T_{7}^{u}\right| \leq C_{8}^{u} \sum_{e \in \Gamma_{h} \cup \partial \Omega_{\mathrm{ver}}} \frac{r_{u}^{2}}{|e|}\left\|\left[\tau^{u}\right]\right\|_{0, e}^{2}+C^{*} \frac{h^{2 \min \left(r_{u}+1, s_{u}\right)-2}}{r_{u}^{2 s_{u}-4}} .
$$

After obtaining the estimates (A.15) and (A.17)-(A.23), we plug them into (A.14) and use the assumption $h<1$ to obtain

$$
\begin{gathered}
\frac{5}{16}\left\|\tau^{u}\right\|_{0, \Omega}^{2}+\left(\sigma_{u}-C_{1}^{u}-C_{4}^{u}-C_{6}^{u}-C_{7}^{u}-C_{8}^{u}\right) \sum_{e \in \Gamma_{h} \cup \partial \Omega_{\mathrm{ver}}} \frac{r_{u}^{2}}{|e|}\left\|\left[\tau^{u}\right]\right\|_{0, e}^{2} \leq\left(1+C_{2}^{u}+C_{5}^{u}\right)\left\|\tau^{c}\right\|_{0, \Omega}^{2} \\
+\frac{1}{2}\|\|\left(\tau^{c}\right)_{x} \mid\left\|_{0, \Omega}^{2}+C_{3}^{u} \sum_{e \in \Gamma_{h}} \frac{r_{c}^{2}}{|e|}\right\|\left[\tau^{c}\right] \|_{0, e}^{2}+C_{u}^{*}\left(\frac{h^{2 \min \left(r_{c}+1, s_{c}\right)-2}}{r_{c}^{2 s_{c}-2}}+\frac{h^{2 \min \left(r_{u}+1, s_{u}\right)-2}}{r_{u}^{2 s_{u}-4}}\right) .
\end{gathered}
$$

In the same way as we have derived the estimate (A.24), we can establish the following bound:

$$
\begin{gathered}
\frac{5}{16}\left\|\tau^{v}\right\|_{0, \Omega}^{2}+\left(\sigma_{v}-C_{1}^{v}-C_{4}^{v}-C_{6}^{v}-C_{7}^{v}-C_{8}^{v}\right) \sum_{e \in \Gamma_{h} \cup \partial \Omega_{\mathrm{hor}}} \frac{r_{v}^{2}}{|e|}\left\|\left[\tau^{v}\right]\right\|_{0, e}^{2} \leq\left(1+C_{2}^{v}+C_{5}^{v}\right)\left\|\tau^{c}\right\|_{0, \Omega}^{2} \\
+\frac{1}{2}\|\|\left(\tau^{c}\right)_{y} \mid\left\|_{0, \Omega}^{2}+C_{3}^{v} \sum_{e \in \Gamma_{h}} \frac{r_{c}^{2}}{|e|}\right\|\left[\tau^{c}\right] \|_{0, e}^{2}+C_{v}^{*}\left(\frac{h^{2 \min \left(r_{c}+1, s_{c}\right)-2}}{r_{c}^{2 s_{c}-4}}+\frac{h^{2 \min \left(r_{v}+1, s_{v}\right)-2}}{r_{v}^{2 s_{v}-4}}\right) .
\end{gathered}
$$


Next, we use Lemma 2.4 to bound $\left\|\tau^{c}\right\|_{0, \Omega}^{2}$ on the RHS of (A.24) and (A.25). This results in

$$
\begin{aligned}
\frac{5}{16}\left\|\tau^{u}\right\|_{0, \Omega}^{2} & +\left(\sigma_{u}-C_{1}^{u}-C_{4}^{u}-C_{6}^{u}-C_{7}^{u}-C_{8}^{u}\right) \sum_{e \in \Gamma_{h} \cup \partial \Omega_{\mathrm{ver}}} \frac{r_{u}^{2}}{|e|}\left\|\left[\tau^{u}\right]\right\|_{0, e}^{2} \\
& \leq\left(\frac{1}{2}+K\left(1+C_{2}^{u}+C_{5}^{u}\right)\right)\|\| \nabla \tau^{c} \mid\left\|_{0, \Omega}^{2}+\left(C_{3}^{u}+K\left(1+C_{2}^{u}+C_{5}^{u}\right)\right) \sum_{e \in \Gamma_{h}} \frac{r_{c}^{2}}{|e|}\right\|\left[\tau^{c}\right] \|_{0, e}^{2} \\
& +C_{u}^{*}\left(\frac{h^{2 \min \left(r_{c}+1, s_{c}\right)-2}}{r_{c}^{2 s_{c}-2}}+\frac{h^{2 \min \left(r_{u}+1, s_{u}\right)-2}}{r_{u}^{2 s_{u}-4}}\right)
\end{aligned}
$$

and

$$
\begin{aligned}
\frac{5}{16}\left\|\tau^{v}\right\|_{0, \Omega}^{2} & +\left(\sigma_{v}-C_{1}^{v}-C_{4}^{v}-C_{6}^{v}-C_{7}^{v}-C_{8}^{v}\right) \sum_{e \in \Gamma_{h} \cup \partial \Omega_{\mathrm{hor}}} \frac{r_{v}^{2}}{|e|}\left\|\left[\tau^{v}\right]\right\|_{0, e}^{2} \\
& \leq\left(\frac{1}{2}+K\left(1+C_{2}^{v}+C_{5}^{v}\right)\right)\|\| \nabla \tau^{c} \mid\left\|_{0, \Omega}^{2}+\left(C_{3}^{v}+K\left(1+C_{2}^{v}+C_{5}^{v}\right)\right) \sum_{e \in \Gamma_{h}} \frac{r_{c}^{2}}{|e|}\right\|\left[\tau^{c}\right] \|_{0, e}^{2} \\
& +C_{v}^{*}\left(\frac{h^{2 \min \left(r_{c}+1, s_{c}\right)-2}}{r_{c}^{2 s_{c}-2}}+\frac{h^{2 \min \left(r_{v}+1, s_{v}\right)-2}}{r_{v}^{2 s_{v}-4}}\right) .
\end{aligned}
$$

We then multiply both sides of (A.26) and (A.27) by $16 / 5$, choose the appropriate penalty parameters $\sigma_{u}$ and $\sigma_{v}$, integrate with respect to time from 0 to $T$, and use the estimate (A.12) to obtain

$$
\begin{aligned}
& \int_{0}^{T}\left(\left\|\tau^{u}\right\|_{0, \Omega}^{2}+\sum_{e \in \Gamma_{h} \cup \partial \Omega_{\mathrm{ver}}} \frac{r_{u}^{2}}{|e|}\left\|\left[\tau^{u}\right]\right\|_{0, e}^{2}\right) \\
& \quad \leq C^{\mathrm{III}}\left(\frac{h^{2 \min \left(r_{\rho}+1, s_{\rho}\right)-2}}{r_{\rho}^{2 s_{\rho}-4}}+\frac{h^{2 \min \left(r_{c}+1, s_{c}\right)-2}}{r_{c}^{2 s_{c}-4}}+\frac{h^{2 \min \left(r_{u}+1, s_{u}\right)-2}}{r_{u}^{2 s_{u}-4}}+\frac{h^{2 \min \left(r_{v}+1, s_{v}\right)-2}}{r_{v}^{2 s_{v}-4}}\right)
\end{aligned}
$$

and

$$
\begin{aligned}
& \int_{0}^{T}\left(\left\|\tau^{v}\right\|_{0, \Omega}^{2}+\sum_{e \in \Gamma_{h} \cup \partial \Omega_{\mathrm{hor}}} \frac{r_{v}^{2}}{|e|}\left\|\left[\tau^{v}\right]\right\|_{0, e}^{2}\right) \\
& \quad \leq C^{\mathrm{IV}}\left(\frac{h^{2 \min \left(r_{\rho}+1, s_{\rho}\right)-2}}{r_{\rho}^{2 s_{\rho}-4}}+\frac{h^{2 \min \left(r_{c}+1, s_{c}\right)-2}}{r_{c}^{2 s_{c}-4}}+\frac{h^{2 \min \left(r_{u}+1, s_{u}\right)-2}}{r_{u}^{2 s_{u}-4}}+\frac{h^{2 \min \left(r_{v}+1, s_{v}\right)-2}}{r_{v}^{2 s_{v}-4}}\right),
\end{aligned}
$$

where $C^{\mathrm{III}}$ and $C^{\mathrm{IV}}$ are constants that depend on $\|\rho\|_{\left(L^{\infty}([0, T]) ; H^{2}(\Omega)\right)},\left\|\rho_{t}\right\|_{\left(L^{\infty}([0, T]) ; L^{2}(\Omega)\right)}$, $\|c\|_{\left(L^{\infty}([0, T]) ; H^{2}(\Omega)\right)},\left\|c_{t}\right\|_{\left(L^{\infty}([0, T]) ; L^{2}(\Omega)\right)},\|u\|_{\left(L^{\infty}([0, T]) ; L^{2}(\Omega)\right)},\|v\|_{\left(L^{\infty}([0, T]) ; L^{2}(\Omega)\right)}$, and $T$ only.

We now estimate the RHS of (A.24) in a different way: we apply the inequality (2.5) and the inverse inequality (2.7), which yield

$$
\begin{aligned}
\frac{5}{16}\left\|\tau^{u}\right\|_{0, \Omega}^{2} & +\left(\sigma_{u}-C_{1}^{u}-C_{4}^{u}-C_{6}^{u}-C_{7}^{u}-C_{7}^{u}\right) \sum_{e \in \Gamma_{h} \cup \partial \Omega_{\mathrm{ver}}} \frac{r_{u}^{2}}{|e|}\left\|\left[\tau^{u}\right]\right\|_{0, e}^{2} \\
& \leq K_{u} \frac{r_{c}^{4}}{h^{2}}\left\|\tau^{c}\right\|_{0, \Omega}^{2}+C_{u}^{*}\left(\frac{h^{2 \min \left(r_{c}+1, s_{c}\right)-2}}{r_{c}^{2 s_{c}-2}}+\frac{h^{2 \min \left(r_{u}+1, s_{u}\right)-2}}{r_{u}^{2 s_{u}-4}}\right) .
\end{aligned}
$$


We then take the supremum over $t$, choose the appropriate penalty parameters $\sigma_{u}$ and $\sigma_{v}$, and use the estimate (A.12) to obtain

$$
\begin{aligned}
& \sup _{[0, T]}\left(\left\|\tau^{u}\right\|_{0, \Omega}^{2}+\sum_{e \in \Gamma_{h} \cup \partial \Omega_{\mathrm{ver}}} \frac{r_{u}^{2}}{|e|}\left\|\left[\tau^{v}\right]\right\|_{0, e}^{2}\right) \\
& \quad \leq C\left(\frac{h^{2 \min \left(r_{\rho}+1, s_{\rho}\right)-4}}{r_{\rho}^{2 s_{\rho}-8}}+\frac{h^{2 \min \left(r_{c}+1, s_{c}\right)-4}}{r_{c}^{2 s_{c}-8}}+\frac{h^{2 \min \left(r_{u}+1, s_{u}\right)-4}}{r_{u}^{2 s_{u}-8}}+\frac{h^{2 \min \left(r_{v}+1, s_{v}\right)-4}}{r_{v}^{2 s_{v}-8}}\right) .
\end{aligned}
$$

Finally, using the assumptions on $r, s$, and $h$, we conclude that

$$
\sup _{[0, T]}\left(\left\|\tau^{u}\right\|_{0, \Omega}^{2}+\sum_{e \in \Gamma_{h} \cup \partial \Omega_{\mathrm{ver}}} \frac{r_{u}^{2}}{|e|}\left\|\left[\tau^{v}\right]\right\|_{0, e}^{2}\right) \leq C_{u}^{*} h^{2}\left(\frac{1}{r_{\rho}^{4}}+\frac{1}{r_{c}^{4}}+\frac{1}{r_{u}^{4}}+\frac{1}{r_{v}^{4}}\right),
$$

where the constant $C_{u}^{*}$ is independent of $h$ and $r$.

The bound on $\tau^{v}$ is obtained similarly:

$$
\sup _{[0, T]}\left(\left\|\tau^{v}\right\|_{0, \Omega}^{2}+\sum_{e \in \Gamma_{h} \cup \partial \Omega_{\mathrm{hor}}} \frac{r_{v}^{2}}{|e|}\left\|\left[\tau^{v}\right]\right\|_{0, e}^{2}\right) \leq C_{v}^{*} h^{2}\left(\frac{1}{r_{\rho}^{4}}+\frac{1}{r_{c}^{4}}+\frac{1}{r_{u}^{4}}+\frac{1}{r_{v}^{4}}\right)
$$

where $C_{v}^{*}$ is independent of $h$ and $r$.

According to the definition on page 9, the estimates (A.28)-(A.29) and (A.30)-(A.31) ensure that $\left(\phi_{L}^{u}, \phi_{L}^{v}\right) \in S$.

Acknowledgment: The research of Y.Epshteyn is based upon work supported by the Center for Nonlinear Analysis (CNA) under the National Science Foundation Grant \# DMS-0635983. The research of A. Kurganov was supported in part by the NSF Grant \# DMS-0610430.

\section{References}

[1] J. Adler, Chemotaxis in bacteria, Ann. Rev. Biochem., 44 (1975), pp. 341-356.

[2] S. Agmon, Lectures on Elliptic Boundary Value Problems, Van Nostrand, Princeton, NJ, 1965.

[3] V. Aizinger, C. Dawson, B. Cockburn, And P. Castillo, Local discontinuous Galerkin methods for contaminant transport, Adv. in Water Res., 24 (2000), pp. 73-87.

[4] D.N. Arnold, An interior penalty finite element method with discontinuous elements, SIAM J. Numer. Anal., 19 (1982), pp. 742-760.

[5] I. BABUŠKA AND M. SuRI, The h-p version of the finite element method with quasiuniform meshes, RAIRO Modél. Math. Anal. Numér., 21 (1987), pp. 199-238.

[6] I. BABUŠKA AND M. SuRI, The optimal convergence rates of the p-version of the finite element method, SIAM J. Numer. Anal., 24 (1987), pp. 750-776.

[7] G.A. Baker, W.N. Jureidini, and O.A. Karakashian, Piecewise solenoidal vector fields and the Stokes problem, SIAM J. Numer. Anal., 27 (1990), pp. 1466-1485. 
[8] J.T. Bonner, The cellular slime molds, Princeton University Press, Princeton, New Jersey, 2nd ed., 1967.

[9] S. Brenner, Poincaré-Friedrichs inequalities for piecewise $H^{1}$ functions, SIAM J. Numer. Anal., 41 (2003), pp. 306-324.

[10] E.O. Budrene And H.C. BerG, Complex patterns formed by motile cells of escherichia coli, Nature, 349 (1991), pp. 630-633.

[11] E.O. Budrene And H.C. Berg, Dynamics of formation of symmetrical patterns by chemotactic bacteria, Nature, 376 (1995), pp. 49-53.

[12] A. Chertock And A. Kurganov, A positivity preserving central-upwind scheme for chemotaxis and haptotaxis models, Numer. Math. submitted.

[13] S. Childress And J.K. Percus, Nonlinear aspects of chemotaxis, Math. Biosc., 56 (1981), pp. $217-237$.

[14] B. Cockburn, G.E. Karniadakis, and C.-W. Shu, eds., First International Symposium on Discontinuous Galerkin Methods, vol. 11 of Lecture Notes in Computational Science and Engineering, Springer-Verlag, 2000.

[15] B. Cockburn And C.-W. Shu., The local discontinuous Galerkin method for convectiondiffusion systems, SIAM J. Numer. Anal., 35 (1998), pp. 2440-2463.

[16] M.H. Cohen And A. Robertson, Wave propagation in the early stages of aggregation of cellular slime molds, J. Theor. Biol., 31 (1971), pp. 101-118.

[17] C. Dawson, E. Kubatko, and J. Westerink, hp Discontinuous Galerkin methods for advection-dominated problems in shallow water, Comp. Meth. Appl. Mech. Eng. to appear.

[18] C. Dawson, S. Sun, And M.F. Wheeler, Compatible algorithms for coupled flow and transport, Comput. Meth. Appl. Mech. Engng, 193 (2004), pp. 2565-2580.

[19] J. Douglas And T. Dupont, Lecture Notes in Physics, vol. 58, Springer-Verlag, 1976, ch. Interior penalty procedures for elliptic and parabolic Galerkin methods.

[20] Y. Epshteyn AND B. RiviÈRe, On the solution of incompressible two-phase flow by a $p$ version discontinuous Galerkin method, Comm. Numer. Methods Engrg., 22 (2006), pp. 741751.

[21] F. Filbet, A finite volume scheme for the Patlak-Keller-Segel chemotaxis model, Numer. Math., 104 (2006), pp. 457-488.

[22] V. Girault, B. Rivière, And M.F. Wheeler, A discontinuous Galerkin method with non-overlapping domain decomposition for the Stokes and Navier-Stokes problems, Math. Comp., 74 (2005), pp. 53-84.

[23] S. Gottlieb, C.-W. Shu, And E. TAdmor, High order time discretization methods with the strong stability property, SIAM Review, 43 (2001), pp. 89-112. 
[24] D.H. Griffel, Applied Functional Analysis, Dover, 2002.

[25] M.A. Herrero and J.J.L. Velázquez, A blow-up mechanism for a chemotaxis model, Ann. Scuola Normale Superiore, 24 (1997), pp. 633-683.

[26] D. Horstmann, From 1970 until now: The Keller-Segel model in chemotaxis and its consequences I, Jahresber. DMV, 105 (2003), pp. 103-165.

[27] D. Horstmann, From 1970 until now: The Keller-Segel model in chemotaxis and its consequences II, Jahresber. DMV, 106 (2004), pp. 51-69.

[28] E.F. Keller And L.A. Segel, Initiation of slime mold aggregation viewed as an instability, J. Theor. Biol., 26 (1970), pp. 399-415.

[29] E.F. Keller And L.A. Segel, Model for chemotaxis, J. Theor. Biol., 30 (1971), pp. 225234.

[30] E.F. Keller And L.A. Segel, Traveling bands of chemotactic bacteria: A theoretical analysis, J. Theor. Biol., 30 (1971), pp. 235-248.

[31] A. Kurganov And C.-T. Lin, On the reduction of numerical dissipation in central-upwind schemes, Commun. Comput. Phys., 2 (2007), pp. 141-163.

[32] A. Kurganov, S. Noelle, and G. Petrova, Semi-discrete central-upwind schemes for hyperbolic conservation laws and Hamilton-Jacobi equations, SIAM J. Sci. Comput., 23 (2001), pp. 707-740.

[33] A. Kurganov and G. Petrova, Central-upwind schemes on triangular grids for hyperbolic systems of conservation laws, Numer. Methods Partial Differential Equations, 21 (2005), pp. 536-552.

[34] A. Marrocco, 2D simulation of chemotaxis bacteria aggregation, M2AN Math. Model. Numer. Anal., 37 (2003), pp. 617-630.

[35] V. Nanjundiah, Chemotaxis, signal relaying and aggregation morphology, J. Theor. Biol., 42 (1973), pp. 63-105.

[36] C. Ortner And E. SüLI, Discontinuous Galerkin finite element approximation of nonlinear second-order elliptic and hyperbolic systems., SIAM J. Numer. Anal., 45 (2007), pp. 13701391.

[37] C.S. Patlak, Random walk with persistence and external bias, Bull. Math: Biophys., 15 (1953), pp. 311-338.

[38] L.M. Prescott, J.P. Harley, and D.A. Klein, Microbiology, Wm. C. Brown Publishers, Chicago, London, 3rd ed., 1996.

[39] B. Rivière, M.F. Wheeler, And V. Girault., A priori error estimates for finite element methods based on discontinuous approximation spaces for elliptic problems, SIAM J. Numer. Anal., 39 (2001), pp. 902-931. 
[40] S. Sun AND M.F. WHEELER, Symmetric and nonsymmetric discontinuous Galerkin methods for reactive transport in porous media, SIAM J. Numer. Anal., 43 (2005), pp. 195-219.

[41] R. Tyson, S.R. Lubkin, And J.D. Murray, A minimal mechanism for bacterial pattern formation, Proc. Roy. Soc. Lond. B, 266 (1999), pp. 299-304.

[42] R. Tyson, L.G. Stern, And R.J. LeVeque, Fractional step methods applied to a chemotaxis model, J. Math. Biol., 41 (2000), pp. 455-475. 